\title{
The $L^{\infty}$-null Controllability of Parabolic Equation with Equivalued Surface Boundary Conditions*
}

\author{
Qi Lü ${ }^{\dagger}$ and Zhongqi Yin
}

\begin{abstract}
In this paper, we obtain the $L^{\infty}$-null controllability of the parabolic equation with equivalued surface boundary conditions in $\Omega \times[0, T]$. The control is supported in the product of an open subset of $\Omega$ and a subset of $[0, T]$ with positive measure. The main result is obtained by the method of Lebeau-Robianno-type iteration, based on a new estimate for partial sum of the eigenfunctions of the elliptic operator with equivalued surface boundary conditions.
\end{abstract}

Key Words. $L^{\infty}$-null controllability, parabolic equation, equivalued surface boundary condition, Lebeau-Robianno-type iteration.

*This work is partially supported by the NSFC under grants 10831007 and 60974035 , by the Grant MTM200803541 of the MICINN, Spain, by Project PI2010-04 of the Basque Government, the ERC Advanced Grant FP7-246775 NUMERIWAVES and the ESF Research Networking Programme OPTPDE.

${ }^{\dagger}$ Basque Center for Applied Mathematics (BCAM), Bizkaia Technology Park, Building 500, 48160, Derio, Basque Country, Spain; and School of Mathematical Sciences, University of Electronic Science and Technology of China, Chengdu 610054, China. e-mail: qlu@bcamath.org.

${ }^{\ddagger}$ School of Mathematics, Sichuan University, Chengdu 610064, China; and Department of Mathematics, Sichuan Normal University, Chengdu 610068, China. e-mail: zhongqiyin@sohu.com. 


\section{Introduction}

Consider the following controlled parabolic equation with equivalued surface boundary conditions:

$$
\begin{cases}y_{t}-\sum_{i, j=1}^{n}\left(a^{i j}(x) y_{x_{i}}\right)_{x_{j}}=f(x, t) \chi_{\omega} \chi_{E}, & \text { in } Q, \\ \left.y\right|_{\Gamma_{1}}=0,\left.y\right|_{\Gamma_{0}}=k(t) \text { (an unknown function) }, & \\ \int_{\Gamma_{0}} \sum_{i, j=1}^{n} a^{i j} y_{x_{i}} \nu_{j} d \Gamma=0, & \text { in } \Omega . \\ y(x, 0)=y_{0}, & \text { in }\end{cases}
$$

Here, $Q=\Omega \times[0, T]$, the time $T>0$ is given, and $\Omega \subset \mathbb{R}^{n}(n \in \mathbb{N})$ is a bounded domain with a $C^{3}$ boundary $\Gamma=\Gamma_{1} \cup \Gamma_{0}$, such that $\overline{\Gamma_{0}} \cap \overline{\Gamma_{1}}=\emptyset$. The coefficients $a^{i j}(x) \in C^{2}(\bar{\Omega})(i, j=1, \cdots, n)$ satisfy that $a^{i j}=a^{j i}$ and, for some positive constant $\Lambda$,

$$
\sum_{i, j=1}^{n} a^{i j} \xi_{i} \xi_{j} \geq \Lambda|\xi|^{2}, \quad \forall(x, \xi) \in \Omega \times \mathbb{R}^{n} .
$$

Let $\omega$ be an arbitrarily given nonempty open subset of $\Omega$ and $E \subset[0, T]$ with positive measure. Denote by $\chi_{\omega}$ the characteristic function of $\omega$, and by $\nu=\left(\nu_{1}, \nu_{2}, \cdots, \nu_{n}\right)$ the unit outward normal vector of $\Omega$. In equation (1.1), $y=y(x, t)$ is the state variable, $y_{0}(\cdot) \in L^{2}(\Omega)$ is the initial datum, $k(\cdot) \in L^{2}(0, T)$ is unknown but determined by the state $y=y(x, t)$ itself, and $f(x, t) \in$ $L^{\infty}\left(0, T ; L^{2}(\Omega)\right)$ is a control function. Thanks to $[2,10]$, it is easy to show that system (1.1) is well-posed in $Y$, where $Y$ is defined by

$$
Y=\left\{y \in C\left([0, T] ; L^{2}(\Omega)\right) \cap L^{2}\left(0, T ; H^{1}(\Omega)\right)|y|_{\Gamma_{1} \times(0, T)}=0, \Gamma_{0} \text { is the equivalued surface of } y\right\} .
$$

System (1.1) is a controlled parabolic equation with equivalued surface boundary condition for which $\left.y\right|_{\Gamma_{0}}(=k(t))$ is a constant for each $t \in(0, T)$ and therefore $\Gamma_{0}$ is said to be the equivalued surface of the state $y=y(x, t)$.

This paper is addressed to establishing the $L^{\infty}$-null controllability for equation (1.1). The controlled equation (1.1) is said to be $L^{\infty}$ - null controllable in $Y$ at time $T$ if for any $y_{0} \in L^{2}(\Omega)$, there is a control $f \in L^{\infty}\left(0, T ; L^{2}(\Omega)\right)$ such that the solution of equation (1.1) with this control satisfies

$$
y(x, T)=0, \quad x \in \Omega .
$$

To our best knowledge, there are only a few papers (published or not) concerning the controllability of the parabolic equation with equivalued surface boundary conditions. In [8], the null controllability was considered but with a technical condition and the insensitizing control problem was described in [9].

Our main result in this paper is the following theorem: 
Theorem 1.1 For any $y_{0} \in L^{2}(\Omega)$, there is control $f \in L^{\infty}\left(0, T ; L^{2}(\Omega)\right)$ such that $y$, which solves (1.1), can be driven by $f$ to zero at time $T$, i.e., $y(x, T)=0$. The control $f$ has the estimate

$$
\|f\|_{L^{\infty}\left(0, T ; L^{2}(\Omega)\right)}^{2} \leq L\left\|y_{0}\right\|_{L^{2}(\Omega)}^{2}
$$

with $L$ a constant independent of $y_{0}$.

The constant $L$ appeared in Theorem 1.1 will be given in Section 3 explicitly. The control in Theorem 1.1 is associated to the set $E \times \omega$, but not as in most published papers depends on the set $(0, T) \times \omega$ for the null controllability of linear parabolic equations. We complete the proof of Theorem 1.1 by using the Lebeau-Robianno-type iteration, according to a special result in the measure theory in [6] and the observability estimate on the partial sums of eigenfunctions of the elliptic operator with equivalued surface boundary conditions (we state this result in Section 2 and give its proof based on two lemmas, which are proven in the appendix.) It is remarkable that we assume only that the boundary of $\Omega$ is $C^{3}$ regular, not $C^{\infty}$ as in [3].

The rest of this paper is organized as follows. In Section 2, we give some preliminaries. In Section 3, we give the proof of Theorem 1.1. Finally, in the Appendix, we give the proofs of the two lemmas based on which the estimate for the eigenfunctions of the elliptic operator with equivalued surface boundary condition is established, which also has independent interest.

\section{Some Preliminaries}

In this section, we give some auxiliary results, which will be used in the proof of Theorem 1.1.

Define an unbounded operator $A$ on $L^{2}(\Omega)$ as follows

$$
\left\{\begin{array}{l}
\mathcal{D}(A)=\left\{u \in H^{2}(\Omega)|u|_{\Gamma_{1}}=0, \int_{\Gamma_{0}} \sum_{i, j=1}^{n} a^{i j} u_{x_{i}} \nu_{j} d \Gamma=0,\left.u\right|_{\Gamma_{0}}=c \text { (unknown) }\right\}, \\
A u=-\sum_{i, j=1}^{n}\left(a^{i j} u_{x_{i}}\right)_{x_{j}}, \quad \forall u \in \mathcal{D}(A) .
\end{array}\right.
$$

Let $\left\{\lambda_{i}\right\}_{i=1}^{\infty}, 0 \leq \lambda_{1} \leq \lambda_{2} \leq \cdots$, be the eigenvalues of $A$ defined in (2.1) and let $\left\{e_{i}\right\}_{i=1}^{\infty}$ be the corresponding eigenfunctions such that $\left\|e_{i}\right\|_{L^{2}(\Omega)}=1(i=1,2,3, \cdots)$, which serves as an orthonormal basis of $L^{2}(\Omega)$. We have an estimate of the eigenfunctions of operator $A$ as follows:

Theorem 2.1 There exist two positive constants $C_{1}, C_{2}$ such that

$$
\sum_{\lambda_{i} \leq r}\left|a_{i}\right|^{2} \leq C_{1} e^{C_{2} \sqrt{r}} \int_{\omega}\left|\sum_{\lambda_{i} \leq r} a_{i} e_{i}\right|^{2} d x
$$

for every finite $r>0$ and every choice of $\left\{a_{i}\right\}_{\lambda_{i} \leq r}$ with $a_{i} \in \mathbb{C}$.

In [3], Lebeau and Zuazua addressed a sketch presentation for the case of Direchlet boundary conditon and based on which they analyzed the null controllability of a linear system of thermoelasticity. As for the case with equivalued surface boundary condition, things are different. Due to the 
special boundary condition, in order to obtain a global Carleman estimate, we need to construct a special corresponding weight function, which plays a crucial role in the proof of obtaining Theorem 2.1.

Proof of Theorem 2.1. First, we introduce two lemmas for the following elliptic equation with equivalued surface boundary conditions:

$$
\begin{cases}u_{t t}+\sum_{i, j=1}^{n}\left(a^{i j} u_{x_{i}}\right)_{x_{j}}=0 & \text { in } Q, \\ \left.u\right|_{\Gamma_{1}}=0, \int_{\Gamma_{0}} \sum_{i, j=1}^{n} a^{i j} u_{x_{i}} \nu_{j} d \Gamma=0,\left.u\right|_{\Gamma_{0}}=c(t) \text { (unknown). } & \end{cases}
$$

Lemma 2.1 Let $0<\gamma<\frac{T}{2}, \gamma<T^{\prime}<T^{\prime \prime}<T-\gamma$, then there exists a constant $\mu \in(0,1)$ such that for any $u \in H^{2}(Q)$, which solves equation (2.3), satisfies

$$
\|u\|_{L^{2}\left(\Omega \times\left(T^{\prime}, T^{\prime \prime}\right)\right)} \leq C\|u\|_{L^{2}(\omega \times(\gamma, T-\gamma))}^{\tau}\|u\|_{H^{1}(Q)}^{1-\tau} .
$$

Lemma 2.2 Let $0<\gamma<\frac{T}{2}$, then there exists a constant $\delta \in(0,1)$ such that for any $u \in H^{2}(Q)$, which solves equation (2.3), satisfies

$$
\|u\|_{H^{1}(\omega \times(\gamma, T-\gamma))} \leq C\left(\|u(0)\|_{L^{2}(\omega)}+\left\|u_{t}(0)\right\|_{L^{2}(\omega)}+\|\nabla u(0)\|_{L^{2}(\omega)}\right)^{\delta}\|u\|_{H^{1}(Q)}^{1-\delta} .
$$

The proofs of these two lemmas is very long, we leave it to the Appendix for simplicity.

Second, we adopt the standard method (see $[3,4])$ to complete the proof. For simplicity of notations, we take $T=4, T^{\prime}=1, T^{\prime \prime}=3$. Following Lemma 2.1 and Lemma 2.2, we have respectively, for $u \in H^{2}(Q)$ solving equation (2.3), that

$$
\|u\|_{L^{2}(\Omega \times(1,3))} \leq C\|u\|_{L^{2}(\omega \times(\gamma, 4-\gamma))}^{\tau}\|u\|_{H^{1}(Q)}^{1-\tau},
$$

and

$$
\|u\|_{H^{1}(\omega \times(\gamma, T-\gamma))} \leq C\left(\|u(0)\|_{L^{2}(\omega)}+\left\|u_{t}(0)\right\|_{L^{2}(\omega)}+\|\nabla u(0)\|_{L^{2}(\omega)}\right)^{\delta}\|u\|_{H^{1}(Q)}^{1-\delta},
$$

which conclude that

$$
\|u\|_{L^{2}(\Omega \times(1,3))} \leq C\left(\|u(0)\|_{L^{2}(\omega)}+\left\|u_{t}(0)\right\|_{L^{2}(\omega)}+\|\nabla u\|_{L^{2}(\omega)}\right)^{\tau \delta}\|u\|_{H^{1}(Q)}^{1-\tau \delta} .
$$

Let $b_{j}=\sqrt{\lambda_{j}}$ and

$$
y(x, t)=\sum_{\lambda_{i} \leq r} \frac{\operatorname{sh} t b_{j}}{b_{j}} a_{j} e_{j},
$$

and $\frac{\operatorname{sh}(t b)}{b}=t$ for $b=0$. It is a straightforward calculation to show that $y$ given as above solves equation (2.3), which vanishes when $(x, t) \in \omega \times\{0\}$. It is obvious that both $\operatorname{Re} y$ and $\operatorname{Im} y$ satisfy (2.8). Applying (2.8) to Re $y$ gives that

$$
\|\operatorname{Re} y\|_{L^{2}\left(\omega_{0} \times(1,3)\right)} \leq C\left\|\operatorname{Re} y_{t}(0)\right\|_{L^{2}(\omega)}^{\tau \delta}\|\operatorname{Re} y\|_{H^{1}(Q)}^{1-\tau \delta} .
$$


Next, we do some estimate on both sides of (2.10). First, we have that

$$
\begin{aligned}
\|\operatorname{Re} y\|_{L^{2}\left(\omega_{0} \times(1,3)\right)}^{2} & =\int_{0}^{3} \int_{\omega_{0}}\left|\sum_{\lambda_{j} \leq r} \frac{\operatorname{sh} t b_{j}}{b_{j}} \operatorname{Re} a_{j} e_{j}\right|^{2} d x d t \\
& =\sum_{\lambda_{j} \leq r}\left|\operatorname{Re} a_{j}\right|^{2} \int_{1}^{3} \int_{\omega_{0}}\left|\frac{\operatorname{sh} t b_{j}}{b_{j}}\right|^{2} d x d t \\
& \geq \sum_{\lambda_{j} \leq r}\left|\operatorname{Re} a_{j}\right|^{2} \int_{1}^{3} t^{2} d t=\frac{8}{3} \sum_{\lambda_{j} \leq r}\left|\operatorname{Re} a_{j}\right|^{2} .
\end{aligned}
$$

Second, for the right hand side of (2.10), we have that

$$
\left\{\begin{array}{l}
\partial_{t} \operatorname{Re} y(x, 0)=\sum_{\lambda_{j} \leq r} \operatorname{Re} a_{j} e_{j}, \\
\|\operatorname{Re} y\|_{H^{1}(Q)}^{2} \leq C^{8 \sqrt{r}}(1+r) \sum_{\lambda_{j} \leq r}\left|\operatorname{Re} a_{j}\right|^{2} \leq C e^{9 \sqrt{r}} \sum_{\lambda_{j} \leq r}\left|\operatorname{Re} a_{j}\right|^{2} .
\end{array}\right.
$$

This together with (2.11) gives that

$$
\sum_{\lambda_{j} \leq r}\left|\operatorname{Re} a_{j}\right|^{2} \leq C_{1} e^{C_{2} \sqrt{r}} \int_{\omega}\left|\sum_{\lambda_{j} \leq r} \operatorname{Re} a_{j} e_{j}\right|^{2} d x .
$$

By the same manner, we have for the imaginary part $\operatorname{Im} y$ that

$$
\sum_{\lambda_{j} \leq r}\left|\operatorname{Im} a_{j}\right|^{2} \leq C_{1} e^{C_{2} \sqrt{r}} \int_{\omega}\left|\sum_{\lambda_{j} \leq r} \operatorname{Im} a_{j} e_{j}\right|^{2} d x .
$$

Combing (2.13) and (2.14), we complete the proof with

$$
\sum_{\lambda_{j} \leq r}\left|a_{j}\right|^{2} \leq C_{1} e^{C_{2} \sqrt{r}} \int_{\omega}\left|\sum_{\lambda_{j} \leq r} a_{j} e_{j}\right|^{2} d x
$$

as in desire.

Let $X_{r}$ be the finite dimensional space spanned by $\left\{e_{i}(x)\right\}_{\lambda_{i} \leq r}$ and $P_{r}: L^{2}(\Omega) \rightarrow X_{r}$ the projection operator from $L^{2}(\Omega)$ to $X_{r}$. In the sequel, the symbol $m(\cdot)$ represents the Lebesgue measure of a measurable set.

Lemma 2.3 For each $r>0$, there corresponds a control $f_{r} \in L^{\infty}\left(0, T ; L^{2}(\Omega)\right)$ with

$$
\left\|f_{r}\right\|_{L^{\infty}\left(0, T ; L^{2}(\Omega)\right)} \leq \frac{C_{1} e^{C_{2} \sqrt{r}}}{|m(E)|^{2}}\left\|y_{0}\right\|_{L^{2}(\Omega)}^{2}
$$

such that $P_{r}(y(\cdot, T))=0$, where $y$ solves system (1.1) with $f=f_{r}$ and $C_{1}, C_{2}$ are two constants appeared in (2.2).

Proof. The idea of the proof is as follows: First, we prove an estimate with respect to $q(x, 0)$, then we deduce the expected result by dual argument and Riesz Representation Theorem. 
Let $q(x, t)$ be the solution of the following equation:

$$
\begin{cases}q_{t}+\sum_{i, j=1}^{n}\left(a^{i j}(x) q_{x_{i}}\right)_{x_{j}}=0, & \text { in } \Omega \times(0, T), \\ \left.q\right|_{\Gamma_{1}}=0,\left.q\right|_{\Gamma_{0}}=c_{1}(t) \text { (an unknown function), } & \text { in }(0, T), \\ \int_{\Gamma_{0}} \sum_{i, j=1}^{n} a^{i j} q_{x_{i}} \nu_{j} d \Gamma=0, & \\ q(x, T) \in X_{r} . & \end{cases}
$$

For that $X_{r}$ is of finite dimension, $q(x, T)$ has representation of the form

$$
q(x, T)=\sum_{\lambda_{i} \leq r} a_{i} e_{i}
$$

for a sequence $\left\{a_{i}\right\}_{\lambda_{i} \leq r}$. It is easy for one to verify that the solution of equation (2.17) has the representation as

$$
q(x, t)=\sum_{\lambda \leq r} a_{i} e^{-\lambda_{i}(T-t)} e_{i}(x), \quad \forall t \in[0, T] .
$$

Then thanks to (2.2), we have that

$$
\begin{aligned}
\int_{\Omega} q^{2}(x, 0) d x & =\sum_{\lambda_{i} \leq r} a_{i}^{2} e^{-2 \lambda_{i} T} \\
& \leq \sum_{\lambda_{i} \leq r}\left|a_{i} e^{-\lambda_{i}(T-t)}\right|^{2} \\
& \leq C_{1} e^{C_{2} \sqrt{r}} \int_{\omega}\left|\sum_{\lambda_{i} \leq r}\left(a_{i} e^{-\lambda_{i}(T-t)}\right) e_{i}\right|^{2} d x \\
& =C_{1} e^{C_{2} \sqrt{r}} \int_{\omega} q^{2}(x, t) d x, \quad \forall t \in[0, T] .
\end{aligned}
$$

As a result, it follows that

$$
\int_{E}\left[\int_{\Omega} q^{2}(x, 0) d x\right]^{\frac{1}{2}} d t \leq\left(C_{1} e^{C_{2} \sqrt{r}}\right)^{\frac{1}{2}} \int_{E}\left[\int_{\omega} q^{2}(x, t) d x\right]^{\frac{1}{2}} d t .
$$

We therefore arrive at the necessary inequality needed later, i.e.,

$$
\begin{aligned}
\int_{\Omega} q^{2}(x, 0) d x & \leq \frac{C_{1} e^{C_{2} \sqrt{r}}}{|m(E)|^{2}}\left\{\int_{0}^{T}\left[\int_{\Omega}\left|\chi_{E} \chi_{\omega} q(x, t)\right|^{2} d x\right]^{\frac{1}{2}}\right\}^{2} \\
& =\frac{C_{1} e^{C_{2} \sqrt{r}}}{|m(E)|^{2}}\left\|\chi_{E} \chi_{\omega} q\right\|_{L^{1}\left(0, T ; L^{2}(\Omega)\right)}^{2} .
\end{aligned}
$$

Next, Let $y(x, t)$ be the solution of system (1.1) and multiply system (1.1) by $q(x, t)$, which solves equation (2.17), then integration by parts gives that

$$
\int_{\Omega} y(x, T) q(x, T) d x-\int_{\Omega} y_{0}(x) q(x, 0) d x=\int_{0}^{T} \int_{\Omega} \chi_{E} \chi_{\omega} f(x, t) q(x, t) d x d t, \quad q(x, T) \in X_{r} .
$$


It is clear that if we can find a $f_{r}(x, t) \in L^{\infty}\left(0, T ; L^{2}(\Omega)\right)$ such that $P_{r}(y(\cdot, T))=0$, then the first term in the above equation can be deserted. In what follows, we are to shows the existence of such control function $f_{r}(x, t)$ with the help of Riesz-type Representation Theorem (See [1, Page 98, Theorem 1]). Define

$$
Y_{r}=\left\{\chi_{E}(t) \chi_{\omega}(x) q(x, t) \mid q(x, t) \text { solves equation }(2.17)\right\}
$$

which is a linear subspace of $L^{1}\left(0, T ; L^{2}(\Omega)\right)$. Define $F_{r}: Y_{r} \rightarrow \mathbb{R}$ by

$$
F_{r}\left(\chi_{E} \chi_{\omega} q\right)=-\int_{\Omega} y_{0}(x) q(x, 0) d x
$$

then following from (2.19) we have that

$$
\left|F_{r}\left(\chi_{E} \chi_{\omega} q\right)\right|^{2} \leq\left\|y_{0}\right\|_{L^{2}(\Omega)}^{2}\|q(x, 0)\|_{L^{2}(\Omega)}^{2} \leq \frac{C_{1} e^{C_{2} \sqrt{r}}}{|m(E)|^{2}}\left\|y_{0}\right\|_{L^{2}(\Omega)}^{2}\left\|\chi_{E} \chi_{\omega} q\right\|_{L^{1}\left(0, T ; L^{2}(\Omega)\right)}^{2},
$$

which tells that $F_{r}$ is a bounded linear functinal on $Y_{r}$. According to the Hahn-Banach theorem, one can extend $F$ to the whole space $L^{1}\left(0, T ; L^{2}(\Omega)\right)$ as a bounded linear functional with norm preserved. We use $F$ to denote this extension. By means of Riesz representation theorem, there must be some $f_{r} \in L^{\infty}\left(0, T ; L^{2}(\Omega)\right)$ satisfying

$$
F(g)=\int_{0}^{T} \int_{\Omega} g f_{r} d x d t, \quad \forall g \in L^{1}\left(0, T ; L^{2}(\Omega)\right)
$$

with

$$
\left\|f_{r}\right\|_{L^{\infty}\left(o, T ; L^{2}(\Omega)\right)}^{2}=\|F\|_{L\left(L\left(0, T ; L^{2}(\Omega)\right) ; \mathbb{R}\right)}^{2} \leq \frac{C_{1} e^{C_{2} \sqrt{r}}}{|m(E)|^{2}}\left\|y_{0}\right\|_{L^{2}(\Omega)}^{2} .
$$

In particular, take $g=\chi_{E} \chi_{\omega} q$ and then we complete the proof.

We also need the following lemma:

Lemma 2.4 [6, Page 256-257] For almost all $\widetilde{t} \in E$, there is a sequence of real numbers $\left\{t_{n}\right\}_{n=1}^{\infty} \subset$ $[0, T]$ with the properties

$$
\begin{aligned}
& \text { (a) } t_{n}<t_{n+1}<\tilde{t} \text { and } t_{n} \rightarrow \widetilde{t} \text { as } n \rightarrow \infty \\
& \text { (b) } m\left(E \cap\left[t_{n}, t_{n+1}\right]\right) \geq \rho\left(t_{n+1}-t_{n}\right) \\
& \text { (c) } \frac{t_{n+1}-t_{n}}{t_{n+2}-t_{n+1}} \leq C_{0}, n=1,2, \cdots
\end{aligned}
$$

where $C_{0}, \rho$ are two positive numbers depending only on the set $E$ itself.

\section{Proof of the Theorem 1.1}

Now we turn to the proof of Theorem 1.1. We use the Lebeau-Robianno-type iteration to do this and borrow some idea from [5] and [7]. 
Proof of Theorem 1.1. To make use of Lemma 2.4, we take $\widetilde{t} \in E$ with $\widetilde{t}<T$ and $\left\{t_{N}\right\}_{N=1}^{\infty} \subseteq$ $(0, T)$ such that $(b)$ and $(c)$ of Lemma 2.4 hold for some $\rho$ and $C_{0}$ and such that

$$
\widetilde{t}-t_{1} \leq \min \left\{1, \lambda_{1}\right\} .
$$

We present

$$
\left\{\begin{array}{l}
{\left[t_{1}, \widetilde{t}\right)=\bigcup_{N=1}^{\infty}\left(I_{N} \cup J_{N}\right),} \\
I_{N}=\left[t_{2 N-1}, t_{2 N}\right], \quad J_{N}=\left[t_{2 N}, t_{2 N+1}\right], \quad N \in \mathbb{N} .
\end{array}\right.
$$

Based on Lemma 2.4, it is clear that $m\left(E \cap I_{N}\right)>0$ for all $N \in \mathbb{N}$.

Step 1. In this step, we prove that for any $\widetilde{y}_{0} \in L^{2}(\Omega)$, there exists a control $\widetilde{f} \in L^{\infty}\left(t_{1}, \widetilde{t} ; L^{2}(\Omega)\right)$ with $\|\widetilde{f}\|_{L^{\infty}\left(t_{1}, \tilde{t} ; L^{2}(\Omega)\right)}^{2} \leq L\|\widetilde{y}\|_{L^{2}(\Omega)}^{2}$, where $L$ is some constant to be determined but independent of $\widetilde{y}_{0}$, so that the solution $\widetilde{y}$ of equation (1.1) satisfying $\widetilde{y}(x, \widetilde{t})=0$ in $L^{2}(\Omega)$, where $\widetilde{y}(x, t)$ solves the following equation:

$$
\begin{cases}\widetilde{y}_{t}-\sum_{i, j=1}^{n}\left(a^{i j}(x) \widetilde{y}_{x_{i}}\right)_{x_{j}}=\widetilde{\chi}_{E} \chi_{\omega} f(x, t), & \text { in } \Omega \times\left(t_{1}, \widetilde{t}\right), \\ \left.\widetilde{y}\right|_{\Gamma_{1}}=0,\left.\widetilde{y}\right|_{\Gamma_{0}}=k(t) \text { (an unknown function), } & t \in\left(t_{1}, \widetilde{t}\right), \\ \int_{\Gamma_{0}} \sum_{i, j=1}^{n} a^{i j} \widetilde{y}_{x_{i}} \nu_{j} d \Gamma=0, & \text { in } \Omega .\end{cases}
$$

We shall verify this claim by induction.

Consider the following two kind of equations:

$$
\begin{cases}y_{t}^{N}-\sum_{i, j=1}^{n}\left(a^{i j}(x) y_{x_{i}}^{N}\right)_{x_{j}}=\chi_{E} \chi_{\omega} f_{N}(x, t), & \text { in } \Omega \times\left(t_{2 N-1}, t_{2 N}\right), \\ \left.y^{N}\right|_{\Gamma_{1}}=0,\left.y^{N}\right|_{\Gamma_{0}}=k(t) \text { (an unknown function), } & \\ \int_{\Gamma_{0}} \sum_{i, j=1}^{n} a^{i j} y_{x_{i}}^{N} \nu_{j} d \Gamma=0, & \text { in } \Omega, \\ y^{N}\left(x, t_{2 N-1}\right)=z^{N-1}\left(x, t_{2 N-1}\right), & \end{cases}
$$

on the interval $I_{N}$, and on the interval $J_{N}$ we have that

$$
\begin{cases}z_{t}^{N}-\sum_{i, j=1}^{n}\left(a^{i j}(x) z_{x_{i}}^{N}\right)_{x_{j}}=0, & \text { in } \Omega \times\left(t_{2 N}, t_{2 N+1}\right), \\ \left.z^{N}\right|_{\Gamma_{1}}=0,\left.z^{N}\right|_{\Gamma_{0}}=k(t) \text { (an unknown function) }, & \\ \int_{\Gamma_{0}} \sum_{i, j=1}^{n} a^{i j} z_{x_{i}}^{N} \nu_{j} d \Gamma=0, & \text { in } \Omega, \\ z^{N}\left(x, t_{2 N}\right)=y^{N}\left(x, t_{2 N}\right), & \end{cases}
$$


with $z_{0}=\widetilde{y}_{0}(x) \in L^{2}(\Omega)$ be given in advance. We will prove by deduction that for each $r_{N}>0$, there exists some certain control $f_{N} \in L^{\infty}\left(I_{N}, ; L^{2}(\Omega)\right)$ satisfying

$$
\begin{aligned}
\left\|y^{N}\left(\cdot, t_{2 N}\right)\right\|_{L^{2}(\Omega)}^{2} & \leq 2^{N}\left(\frac{C_{1}}{\rho^{2}\left(t_{2}-t_{1}\right)^{2}}\right)^{N} C_{0}^{4 \frac{N(N-1)}{2}} \prod_{i=1}^{N} \alpha_{i}\left\|\widetilde{y}_{0}\right\|_{L^{2}(\Omega)}^{2}, \\
\left\|z^{N}\left(\cdot, t_{2 N+1}\right)\right\|_{L^{2}(\Omega)}^{2} & \leq e^{-2 r_{N}\left(t_{2 N+1}-t_{2 N}\right)}\left\|y^{N}\left(x, t_{2 N}\right)\right\|_{L^{2}(\Omega)}^{2}, \\
\left\|f_{N}\right\|_{L^{\infty}\left(I_{N} ; L^{2}(\Omega)\right)}^{2} & \leq 2^{N-1}\left(\frac{C_{1}}{\rho^{2}\left(t_{2}-t_{1}\right)^{2}}\right)^{N} C_{0}^{4\left(\frac{N(N-1)}{2}\right)} \prod_{k=1}^{N} \alpha_{k}\left\|\widetilde{y}_{0}\right\|_{L^{2}(\Omega)}^{2} \\
& =\widetilde{C}^{N(N-1)} \prod_{k=1}^{N} \alpha_{k}\left\|\widetilde{y}_{0}\right\|_{L^{2}(\Omega)}^{2}
\end{aligned}
$$

with

$$
\alpha_{N}= \begin{cases}e^{C_{2} \sqrt{r_{1}}}, & N=1 \\ e^{C_{2} \sqrt{r_{N}}} e^{-2 r_{N-1}\left(t_{3}-t_{2}\right) C_{0}^{-2(N-2)}}, & N \geq 2\end{cases}
$$

and such that $P_{r_{N}}\left(y_{N}\left(\cdot, t_{2 N}\right)\right)=0$ and $\widetilde{C}=\frac{2 C_{1}}{\rho^{2}\left(t_{2}-t_{1}\right)^{2}} C_{0}^{2}$.

In what follows, we do this step by step. First, consider on the time interval $I_{1}=\left[t_{1}, t_{2}\right]$ the following controlled parabolic equation

$$
\begin{cases}y_{t}^{1}-\sum_{i, j=1}^{n}\left(a^{i j}(x) y_{x_{i}}^{1}\right)_{x_{j}}=f^{1}(x, t) \chi_{\omega} \chi_{E}, & \text { in } \Omega \times\left(t_{1}, t_{2}\right), \\ \left.y^{1}\right|_{\Gamma_{1}}=0,\left.y^{1}\right|_{\Gamma_{0}}=k(t) \text { (an unknown function), } & \\ \int_{\Gamma_{0}} \sum_{i, j=1}^{n} a^{i j} y_{x_{i}}^{1} \nu_{j} d s=0, & \text { in } \Omega, \\ y^{1}\left(x, t_{1}\right)=\widetilde{y}_{0}, & \end{cases}
$$

Making use of Lemma 2.3, for any $r_{1}>0$, there is a control $f_{1} \in L^{\infty}\left(t_{1}, t_{2} ; L^{2}(\Omega)\right)$ with the property

$$
\left\|f_{1}\right\|_{L^{\infty}\left(t_{1}, t_{2} ; L^{2}(\Omega)\right)}^{2} \leq \frac{C_{1} e^{C_{2} \sqrt{r_{1}}}}{\left|m\left(E \cap\left[t_{1}, t_{2}\right]\right)\right|^{2}}\left\|\widetilde{y}_{0}\right\|_{L^{2}(\Omega)}^{2}
$$

such that $P_{r_{1}}\left(y_{1}\left(\cdot, t_{2}\right)\right)=0$. Then by $(b)$ and $(c)$ of $(2.20)$ in Lemma 2.4, one has that

$$
\left\|f_{1}\right\|_{L^{\infty}\left(t_{1}, t_{2} ; L^{2}(\Omega)\right)}^{2} \leq \frac{C_{1} e^{C_{2} \sqrt{r_{1}}}}{\rho^{2}\left(t_{2}-t_{1}\right)^{2}}\left\|\widetilde{y}_{0}\right\|_{L^{2}(\Omega)}^{2}=\frac{C_{1}}{\rho^{2}\left(t_{2}-t_{1}\right)^{2}} \alpha_{1}\left\|\widetilde{y}_{0}\right\|_{L^{2}(\Omega)}^{2}
$$

by letting $\alpha_{1}=e^{C_{2} \sqrt{r_{1}}}$. Furthermore, multiplying (3.5) by $y_{1}$ and integration by parts shows that

$$
\frac{d}{d t}\left\|y^{1}(\cdot, t)\right\|_{L^{2}(\Omega)}^{2} \leq-2 \lambda_{1}\left\|y^{1}(\cdot, t)\right\|_{L^{2}(\Omega)}^{2}+2 \int_{\Omega} y^{1}(x, t) \chi_{E} \chi_{\omega} f_{1}(x, t) d x .
$$


Integrating this equality from $t_{1}$ to $t_{2}$ with respect to the time variable $t$, one finds that

$$
\begin{aligned}
\left\|y^{1}\left(\cdot, t_{2}\right)\right\|_{L^{2}(\Omega)}^{2} \leq & \left\|y^{1}\left(t_{1}\right)\right\|_{L^{2}(\Omega)}^{2}-2 \lambda_{1} \int_{t_{1}}^{t_{2}}\left\|y^{1}(t)\right\|_{L^{2}(\Omega)}^{2} d t \\
& +2 \int_{t_{1}}^{t_{2}} \int_{\Omega} y_{1}(x, t) \chi_{E} \chi_{\omega} f_{1}(x, t) d x d t \\
\leq & \left\|y^{1}\left(\cdot, t_{1}\right)\right\|_{L^{2}(\Omega)}^{2}-2 \lambda_{1} \int_{t_{1}}^{t_{2}}\left\|y^{1}(t)\right\|_{L^{2}(\Omega)}^{2} d t+\lambda_{1} \int_{t_{1}}^{t_{2}}\left\|y^{1}(t)\right\|_{L^{2}(\Omega)}^{2} d x \\
& +\frac{t_{2}-t_{1}}{\lambda_{1}}\left\|f_{1}\right\|_{L^{\infty}\left(t_{1}, t_{2} ; L^{2}(\Omega)\right)}^{2} \\
\leq & \left\|\widetilde{y}_{0}\right\|_{L^{2}(\Omega)}^{2}+\frac{t_{2}-t_{1}}{\lambda_{1}}\left\|f_{1}\right\|_{L^{\infty}\left(t_{1}, t_{2} ; L^{2}(\Omega)\right)}^{2} \\
\leq & 2 \frac{C_{1}}{\rho^{2}\left(t_{2}-t_{1}\right)^{2}} \alpha_{1}\left\|\widetilde{y}_{0}\right\|_{L^{2}(\Omega)}^{2} .
\end{aligned}
$$

On the other hand, on the interval $J_{1}=\left[t_{3}, t_{4}\right]$, we consider the following equation but without control:

$$
\begin{cases}z_{t}^{1}-\sum_{i, j=1}^{n}\left(a^{i j}(x) z_{x_{i}}^{1}\right)_{x_{j}}=0, & \text { in } \Omega \times\left(t_{2}, t_{3}\right), \\ \left.z^{1}\right|_{\Gamma_{1}}=0,\left.z^{1}\right|_{\Gamma_{0}}=k(t) \text { (an unknown function) }, & \\ \int_{\Gamma_{0}} \sum_{i, j=1}^{n} a^{i j} z_{x_{i}}^{1} \nu_{j} d \Gamma=0, & \text { in } \Omega . \\ z^{1}\left(x, t_{3}\right)=\widetilde{y}^{1}\left(x, t_{2}\right), & \end{cases}
$$

Recalling that $P_{r_{1}}\left(y\left(\cdot, t_{2}\right)\right)=0$, we have that

$$
\frac{d}{d t}\left\|z^{1}(\cdot, t)\right\|_{L^{2}(\Omega)}^{2} \leq-2\left\|\sum_{i, j=1}^{n} a^{i j} z_{x_{i}}^{1}(\cdot, t) z_{x_{j}}^{1}(\cdot, t)\right\|_{L^{2}(\Omega)}^{2} \leq-2 r_{1}\left\|z_{1}(\cdot, t)\right\|_{L^{2}(\Omega)}^{2} .
$$

Utilizing Gronwall inequality, we obtain that

$$
\begin{aligned}
\left\|z^{1}\left(\cdot, t_{3}\right)\right\|_{L^{2}(\Omega)}^{2} & \leq e^{-2 r_{1}\left(t_{3}-t_{2}\right)}\left\|y_{1}\left(\cdot, t_{2}\right)\right\|_{L^{2}(\Omega)}^{2} \\
& \leq 2 \frac{C_{1}}{\rho^{2}\left(t_{2}-t_{1}\right)^{2}} \alpha_{1} e^{-2 r_{1}\left(t_{3}-t_{2}\right)}\left\|\widetilde{y}_{0}\right\|_{L^{2}(\Omega)}^{2} .
\end{aligned}
$$

For $I_{2}=\left[t_{3}, t_{4}\right]$, we consider the following controlled equation:

$$
\begin{cases}y_{t}^{2}-\sum_{i, j=1}^{n}\left(a^{i j}(x) y_{x_{i}}^{2}\right)_{x_{j}}=f_{2}(x, t) \chi_{\omega} \chi_{E}, & \text { in } \Omega \times\left(t_{3}, t_{4}\right), \\ \left.y^{2}\right|_{\Gamma_{1}}=0,\left.y^{2}\right|_{\Gamma_{0}}=k(t) \text { (an unknown function) }, & \\ \int_{\Gamma_{0}} \sum_{i, j=1}^{n} a^{i j} y_{x_{i}}^{2} \nu_{j} d \Gamma=0, & \text { in } \Omega . \\ y^{2}\left(x, t_{3}\right)=z^{1}\left(x, t_{3}\right), & \end{cases}
$$


With the similar argument to that for $I_{1}=\left[t_{1}, t_{2}\right]$, there exists a control $f_{2} \in L^{\infty}\left(t_{3}, t_{4} ; L^{2}(\Omega)\right)$ which having the estimate

$$
\begin{aligned}
\left\|f_{2}\right\|_{L^{\infty}\left(t_{3}, t_{4} ; L^{2}(\Omega)\right)}^{2} & \leq \frac{C_{1} e^{C_{2} \sqrt{r_{2}}}}{\left|m\left(E \cap\left[t_{3}, t_{4}\right]\right)\right|^{2}}\left\|z^{1}\left(\cdot, t_{3}\right)\right\|_{L^{2}(\Omega)}^{2} \\
& \leq 2\left(\frac{C_{1}}{\rho^{2}\left(t_{2}-t_{1}\right)^{2}}\right)^{2} C_{0}^{4} \alpha_{1} \alpha_{2}\left\|\widetilde{y}_{0}\right\|_{L^{2}(\Omega)}^{2}
\end{aligned}
$$

with $\alpha_{2}=e^{C_{2} \sqrt{r_{2}}} e^{-2 r_{1}\left(t_{3}-t_{2}\right)}$ and $P_{r_{2}}\left(y_{2}\left(\cdot, t_{4}\right)\right)=0$. Furthermore, as the argument on the interval $I_{1}$, we have estimate for $y^{2}(x, t)$ that

$$
\begin{aligned}
\left\|y^{2}\left(\cdot, t_{4}\right)\right\|_{L^{2}(\Omega)}^{2} \leq & \left\|y^{2}\left(\cdot, t_{3}\right)\right\|_{L^{2}(\Omega)}^{2}-2 \lambda_{1} \int_{t_{3}}^{t_{4}}\left\|y^{2}(\cdot, t)\right\|_{L^{2}(\Omega)}^{2} d t \\
& +2 \int_{t_{3}}^{t_{4}} \int_{\Omega} y^{2}(x, t) \chi_{E} \chi_{\omega} f_{2}(x, t) d x d t \\
\leq & \left\|z^{1}\left(\cdot, t_{3}\right)\right\|_{L^{2}(\Omega)}^{2}-2 \lambda_{1} \int_{t_{3}}^{t_{4}}\left\|y^{2}(\cdot, t)\right\|_{L^{2}(\Omega)}^{2} d t+\lambda_{1} \int_{t_{3}}^{t_{4}} \int_{\Omega}\left|y^{2}(x, t)\right|^{2} d x \\
& +\frac{t_{4}-t_{3}}{\lambda_{1}}\|u\|_{L^{\infty}\left(t_{3}, t_{4} ; L^{2}(\Omega)\right)}^{2} \\
\leq & \left\|z^{1}\left(\cdot, t_{3}\right)\right\|_{L^{2}(\Omega)}^{2}+\frac{t_{4}-t_{3}}{\lambda_{1}}\left\|f_{2}\right\|_{L^{\infty}\left(t_{3}, t_{4} ; L^{2}(\Omega)\right)}^{2} \\
\leq & 2^{2}\left(\frac{C_{1}}{\rho^{2}\left(t_{4}-t_{3}\right)^{2}}\right) C_{0}^{4} \alpha_{1} \alpha_{2}\left\|\widetilde{y}_{0}\right\|_{L^{2}(\Omega)}^{2} .
\end{aligned}
$$

Thus, we proved the cases for $N=1,2$. Now suppose that we had proved the case for $N$, we consider the case for $N+1$.

Consider

$$
\begin{cases}y_{t}^{N+1}-\sum_{i, j=1}^{n}\left(a^{i j}(x) y_{x_{i}}^{N+1}\right)_{x_{j}}=f_{N+1}(x, t) \chi_{E} \chi_{\omega}, & \text { in } \Omega \times\left(t_{2 N+1}, t_{2 N+2}\right), \\ \left.y_{N+1}\right|_{\Gamma_{1}}=0,\left.y_{N+1}\right|_{\Gamma_{0}}=k(t)(\text { an unknown function }), & \\ \int_{\Gamma_{0}} \sum_{i, j=1}^{n} a^{i j} y_{x_{i}}^{N+1} \nu_{j} d \Gamma=0, & \text { in } \Omega, \\ y^{N+1}\left(x, t_{2 N+1}\right)=z^{N}\left(x, t_{2 N+1}\right), & \end{cases}
$$

on the interval $I_{N+1}$ and

$$
\begin{cases}z_{t}^{N+1}-\sum_{i, j=1}^{n}\left(a^{i j}(x) z_{x_{i}}^{N+1}\right)_{x_{j}}=0, & \text { in } \Omega \times\left(t_{2 N+2}, t_{2 N+3}\right), \\ \left.z^{N+1}\right|_{\Gamma_{1}}=0,\left.z^{N+1}\right|_{\Gamma_{0}}=k(t) \text { (an unknown function) }, & \\ \int_{\Gamma_{0}} \sum_{i, j=1}^{n} a^{i j} z_{x_{i}}^{N+1} \nu_{j} d \Gamma=0, & \text { in } \Omega, \\ z^{N+1}\left(x, t_{2 N+2}\right)=y^{N}\left(x, t_{2 N+2}\right), & \end{cases}
$$


on the interval $J_{N+1}$.

First, by Lemma 2.3, we have that there exists $f_{N+1} \in L^{\infty}\left(t_{2 N+1}, t_{2 N+2} ; L^{2}(\Omega)\right)$ such that $P_{r_{N+1}}\left(y\left(\cdot, t_{2 N+2}\right)\right)=0$ and that $f_{N+1}$ satisfies the following estimate

$$
\begin{aligned}
& \left\|f_{N+1}\right\|_{L^{\infty}\left(t_{2 N+1}, t_{2(N+1)} ; L^{2}(\Omega)\right)}^{2} \\
\leq & \frac{C_{1} e^{C_{2} \sqrt{r_{N+1}}}}{\left|m\left(E \cap\left[t_{2 N+1}, t_{2 N+2}\right]\right)\right|^{2}}\left\|z^{N}\left(\cdot, t_{2 N+1}\right)\right\|_{L^{2}(\Omega)}^{2} \\
\leq & \frac{C_{1} e^{C_{2} \sqrt{r_{N+1}}}}{\rho^{2}\left(t_{2 N+2}-t_{2 N+1}\right)^{2}} e^{-2 r_{N}\left(t_{2 N+1}-t_{2 N}\right)}\left\|y^{N}\left(x, t_{2 N}\right)\right\|_{L^{2}(\Omega)}^{2} \\
\leq & \frac{C_{1} e^{C_{2} \sqrt{r_{N+1}}}}{\rho^{2}\left(t_{2 N+2}-t_{2 N+1}\right)^{2}} e^{-2 r_{N}\left(t_{2 N+1}-t_{2 N}\right)} 2^{N}\left(\frac{C_{1}}{\rho^{2}\left(t_{2}-t_{1}\right)^{2}}\right)^{N} C_{0}^{4 \frac{N(N-1)}{2}} \prod_{i=1}^{N} \alpha_{i}\left\|\widetilde{y}_{0}\right\|_{L^{2}(\Omega)}^{2} \\
\leq & 2^{N}\left(\frac{C_{1}}{\rho^{2}\left(t_{2}-t_{1}\right)^{2}}\right)^{N+1} C_{0}^{4 \frac{N(N-1)}{2}} C_{0}^{4 N} e^{C_{2} \sqrt{r_{N+1}}} e^{-2 r_{N}\left(t_{2 N+1}-t_{2 N}\right)} \prod_{i=1}^{N} \alpha_{i}\left\|\widetilde{y}_{0}\right\|_{L^{2}(\Omega)}^{2} \\
\leq & 2^{N}\left(\frac{C_{1}}{\rho^{2}\left(t_{2}-t_{1}\right)^{2}}\right)^{N+1} C_{0}^{4 \frac{(N+1) N}{2}} \prod_{i=1}^{N+1} \alpha_{i}\left\|\widetilde{y}_{0}\right\|_{L^{2}(\Omega)}^{2}
\end{aligned}
$$

with $\alpha_{n+1}=e^{C_{2} \sqrt{r_{N+1}}} e^{-2 r_{N}\left(t_{3}-t_{2}\right) C_{0}^{-2(N-1)}}$.

Then similar to the argument for $N=1$, we have that

$$
\begin{aligned}
& \left\|y^{N+1}\left(\cdot, t_{2(N+2)}\right)\right\|_{L^{2}(\Omega)}^{2} \\
\leq & \left\|y^{N+1}\left(\cdot, t_{2 N+1}\right)\right\|_{L^{2}(\Omega)}^{2}-2 \lambda_{1} \int_{t_{2 N+1}}^{t_{2(N+1)}}\left\|y^{N+1}(\cdot, t)\right\|_{L^{2}(\Omega)}^{2} d t \\
& +2 \int_{t_{2 N+1}}^{t_{2(N+1)}} \int_{\Omega} y^{N+1}(x, t) \chi_{E} \chi_{\omega} f_{N+1}(x, t) d x d t \\
\leq & \left\|y^{N+1}\left(\cdot, t_{2 N+1}\right)\right\|_{L^{2}(\Omega)}^{2}-2 \lambda_{1} \int_{t_{2 N+1}}^{t_{2(N+1)}}\left\|y^{N+1}(\cdot, t)\right\|_{L^{2}(\Omega)}^{2} d t+\lambda_{1} \int_{t_{2(N+1)}}^{t_{2 N+1}}\left\|y^{N+1}(x, t)\right\|_{L^{2}(\Omega)}^{2} d x \\
& +\frac{t_{2(N+1)}-t_{2 N+1}}{\lambda_{1}}\left\|f_{N+1}\right\|_{L^{\infty}\left(t_{1}, t_{2} ; L^{2}(\Omega)\right)}^{2} \\
\leq & \left\|y^{N+1}\left(\cdot, t_{2 N+1}\right)\right\|_{L^{2}(\Omega)}^{2}+\frac{t_{2(N+1)}-t_{2 N+1}}{\lambda_{1}}\left\|f_{N+1}\right\|_{L^{\infty}\left(t_{1}, t_{2} ; L^{2}(\Omega)\right)}^{2} \\
\leq & 2^{N}\left(\frac{C_{1}}{\rho^{2}\left(t_{2}-t_{1}\right)^{2}}\right)^{N} C_{0}^{4} \frac{N(N+1)}{2} \prod_{i=1}^{N+1} \alpha_{i}\left\|\widetilde{y}_{0}\right\|_{L^{2}(\Omega)}^{2} \cdot
\end{aligned}
$$

By means of $P_{r_{N+1}}\left(y\left(\cdot, t_{2 N+2}\right)\right)=0$, utilizing the energy decay of the solution to equation (3.7), we have easily get that

$$
\left\|z^{N+1}\left(x, t_{2 N+3}\right)\right\|_{L^{2}(\Omega)}^{2} \leq e^{-2 r_{N+1}\left(t_{2 N+3}-t_{2 N+1}\right)}\left\|y^{N}\left(x, t_{2 N+2}\right)\right\|_{L^{2}(\Omega)}^{2}
$$


Therefore, we proved the claim by deduction.

In what follows, we choose suitable $L$ such that $\left\|f_{N}\right\|_{L^{\infty}\left(I_{N} ; L^{2}(\Omega)\right)}^{2} \leq L\left\|\widetilde{y}_{0}\right\|_{L^{2}(\Omega)}^{2}$. To this end, we let

$$
r_{N}=\left(\frac{2 \widetilde{C}^{N-1}}{t_{3}-t_{2}}\right)^{4} \equiv\left(A \widetilde{C}^{N-1}\right)^{4}, \quad N \geq 1 .
$$

Noticing that $\widetilde{C}>C_{0}^{2}>1$ and $t_{3}-t_{2}<1$, we obtain that

$$
2^{4}<r_{1}<r_{2}<\cdots<r_{N}<r_{N+1}<\cdots \quad \text { and } r_{N} \rightarrow \infty \text { as } N \rightarrow \infty
$$

and that

$$
r_{N-1}^{\frac{1}{4}}\left(t_{3}-t_{2}\right) C_{0}^{-2(N-2)} \geq 2, \quad N \geq 2 .
$$

As a result, it follows that

$$
e^{-2 r_{N-1}\left(t_{3}-t_{2}\right) C_{0}^{-2(N-2)}} \leq e^{-4 r_{N-1}^{\frac{3}{4}}}, \quad N \geq 2 .
$$

From that

$$
\begin{aligned}
\widetilde{C}^{N(N-1) e^{-r_{N}^{\frac{3}{4}}}} & =\frac{\widetilde{C}^{N(N-1)}}{\left(e^{\frac{1}{4}} r^{\frac{1}{2}}\right)^{r_{N-1}^{2}}} \leq \frac{\widetilde{C}^{N(N-1)}}{\left(e^{\left.2 \widetilde{C}^{(N-1)}\right)^{r_{N-1}^{\frac{1}{2}}}}\right.} \\
& \leq \frac{\widetilde{C}^{N(N-1)}}{\widetilde{C}^{2(N-1) r_{N-1}^{\frac{1}{2}}}}, \quad N \geq 2,
\end{aligned}
$$

we get from the definition of $r_{N}$ that there exists a $N_{1} \in \mathbb{N}$ with $N_{1} \geq 2$ such that

$$
\widetilde{C}^{N(N-1)} e^{-r_{N-1}^{\frac{3}{4}}} \leq 1, \quad N \geq N_{1} .
$$

Again, by the definition of $r_{N}$, one finds that

$$
e^{C_{2} \sqrt{r_{N}}} e^{-r_{N-1}^{\frac{3}{4}}}=e^{C_{2} A^{2} \widetilde{C}^{2(N-1)}} e^{-A^{3} \widetilde{C}^{3(N-2)}}, \quad N \geq 2 .
$$

As a result, there is a natural number $N_{2} \geq 2$ such that

$$
e^{C_{2} \sqrt{r_{N}}} e^{-r_{N-1}^{\frac{3}{4}}} \leq 1, \quad N \geq N_{2} .
$$

Next, Let

$$
N_{0}=\max \left\{N_{1}, N_{2}\right\}
$$

It is easy for one to verify that

$$
\alpha_{N} \leq 1, \quad N \geq N_{0},
$$

and from (3.9), (3.10) and (3.11) that

$$
\begin{aligned}
\widetilde{C}^{N(N-1)} \alpha_{N} & =\widetilde{C}^{N(N-1)} e^{C_{2} \sqrt{r_{N}}} e^{-2 r_{N-1}\left(t_{3}-t_{2}\right) C_{0}^{-2(N-2)}} \\
& \leq \widetilde{C}^{N(N-1)} e^{C_{2} \sqrt{r_{N}}} e^{-4 r_{N-1}^{\frac{3}{4}}} \\
& \leq e^{-2 r_{N-1}^{\frac{3}{4}}} .
\end{aligned}
$$


Now, we let

$$
L=\max \left\{\widetilde{C}^{N(N-1)} \prod_{i=1}^{N} \alpha_{i}, 1 \leq N \leq N_{0}\right\} .
$$

Thus, we proved that

$$
\left\|f_{N}\right\|_{L^{\infty}\left(I_{N} ; L^{2}(\Omega)\right)}^{2} \leq L\left\|\widetilde{y}_{0}\right\|_{L^{2}(\Omega)}^{2} .
$$

Furthermore, we take the control $\tilde{f}$ to be such that

$$
\widetilde{f}(x, t)= \begin{cases}f_{N}(x, t), & x \in \Omega, t \in I_{N}, N \geq 1, \\ 0, & x \in \Omega, t \in J_{N}, N \geq 1 .\end{cases}
$$

Now, let $\widetilde{y}$ be the solution of equation (3.1), then from the argument before, it is easy to see that $\widetilde{y}(\cdot, t)=y^{N}(\cdot, t)$ on $I_{N}$. Again, noting that $P_{r_{N}}\left(y^{N}\left(\cdot, t_{2 N}\right)\right)=0$ holding for $N \geq 1$ and $\left\{r_{N}\right\}_{N=1}^{\infty}$ is strictly increasing, together with the construction of $\widetilde{f}$, we conclude that

$$
P_{r_{N}}\left(\widetilde{y}\left(\cdot, t_{2 M}\right)\right)=0, \quad M \geq N
$$

Since $t_{2 M} \rightarrow \widetilde{t}$ as $M \rightarrow \infty$, we can also obtain that

$$
\widetilde{y}\left(\cdot, t_{2 M}\right) \rightarrow \widetilde{y}(\cdot, \widetilde{t}) \quad \text { in } L^{2}(\Omega) \text { as } M \rightarrow \infty .
$$

These two results tells that $P_{r_{N}}(\widetilde{y}(\cdot, \widetilde{t}))=0$ holding for all $N \geq 1$. It follows that $\widetilde{y}(\cdot, \tilde{t})=0$ for that $r_{N} \rightarrow \infty$ as $N \rightarrow \infty$.

Until now, we have proved that there exists a control $\widetilde{f} \in L^{\infty}\left(t_{1}, \widetilde{t} ; L^{2}(\Omega)\right)$ with the estimate $\|\widetilde{f}\|_{L^{\infty}\left(t_{1}, \tilde{t} ; L^{2}(\Omega)\right)}^{2} \leq L\left\|\widetilde{y}_{0}\right\|_{L^{2}(\Omega)}^{2}$, where $L$ is claimed as before, such that $\widetilde{y}$, which solves (3.1), vanishes at $\tilde{t}$, in other words, $\widetilde{y}(x, \widetilde{t})=0$ in $\Omega$.

Step 2. We complete the proof in this step. To this end, we specify $\widetilde{y}_{0}(x)$. Let $\psi$ be the solution of

$$
\begin{cases}\psi_{t}-\sum_{i, j=1}^{n}\left(a^{i j}(x) \psi_{x_{i}}\right)_{x_{j}}=0, & \text { in } \Omega \times\left(0, t_{1}\right), \\ \left.\psi\right|_{\Gamma_{1}}=0,\left.\psi\right|_{\Gamma_{0}}=k(t)(\text { an unknown function }), & t \in\left(0, t_{1}\right), \\ \int_{\Gamma_{0}} \sum_{i, j=1}^{n} a^{i j} \psi_{x_{i}} \nu_{j} d \Gamma=0, & \\ \psi(x, 0)=y_{0}(x), & \text { in } \Omega .\end{cases}
$$

Let $\widetilde{y}_{0}(x)=\psi\left(x, t_{1}\right)$ and set

$$
f(x, t)= \begin{cases}0, & (x, t) \in \Omega \times\left(0, t_{1}\right), \\ \widetilde{f}(x, t), & (x, t) \in\left(t_{1}, \widetilde{t}\right), \\ 0, & (x, t) \in \Omega \times(t, T) .\end{cases}
$$


It is easy for one to verify that $f$ given as above lies in $L^{\infty}\left(0, T ; L^{2}(\Omega)\right)$, which drives the solution $y$ of (1.1) to zero at time $T$. That is, $y(x, T)=0, x \in \Omega$ and $f$ has the estimate same to $\widetilde{f}$, i.e.,

$$
\|f\|_{L^{\infty}\left(0, T ; L^{2}(\Omega)\right)}^{2} \leq L\left\|y_{0}\right\|_{L^{2}(\Omega)}^{2}
$$

with $L$ claimed as before.

\section{Appendix}

In this appendix, we give a proof of Lemmas 2.1-2.2.

Let $m \in \mathbb{N}$, for any $\varphi \in C^{2}\left(\mathbb{R}^{m}\right)$ and positive numbers $\lambda$ and $\mu$, let

$$
\alpha=e^{\mu \varphi}, \quad \theta=e^{\lambda \alpha} .
$$

Assume that $\left(b^{i j}\right)_{1 \leq i, j \leq m}$ is a symmetric matrix with entries $b^{i j} \in C^{1}\left(\mathbb{R}^{m}\right), i, j=1,2, \cdots, m$. We first recall the following result, whose proof can be found in [4].

Lemma 4.1 Assume that $v \in C^{2}\left(\mathbb{R}^{m}\right)$. Let $w=\theta v$, then we have the following point-wise estimate:

$$
\begin{aligned}
& \theta^{2}\left|\sum_{i, j=1}^{m} b^{i j} v_{x_{i} x_{j}}\right|^{2}+2 \lambda \mu \alpha \sum_{i, j=1}^{m}[ 2 \mu \sum_{k, \ell=1}^{m} b^{k \ell} \varphi_{x_{k}} \varphi_{x_{\ell}} b^{i j} w_{x_{i}} w+\lambda^{2} \mu^{2} \alpha^{2} \sum_{k, \ell=1}^{m} b^{k \ell} \varphi_{k} \varphi_{\ell} b^{i j} \varphi_{x_{i}} w^{2} \\
&\left.+2 \sum_{k, \ell=1}^{m} b^{k \ell} \varphi_{x_{k}} w_{\ell} b^{i j} w_{x_{i}}-\sum_{k, \ell=1}^{m} b^{k \ell} w_{x_{k}} w_{x_{\ell}} b^{i j} \varphi_{x_{i}}\right]_{x_{j}} \\
& \geq \sum_{i, j=1}^{m} c^{i j} w_{x_{i}} w_{x_{j}}+B w^{2}+4 \lambda \mu^{2} \sum_{i, j=1}^{m}\left[\alpha\left(\sum_{k, \ell=1}^{m} b^{k \ell} \varphi_{x_{k}} \varphi_{x_{\ell}}\right) b^{i j}\right]_{x_{j}} w_{x_{i}} w \\
& \quad+4 \lambda \mu \alpha \sum_{i, j=1}^{m} \sum_{k, \ell=1}^{m}\left(b^{k \ell} \varphi_{x_{k}} b^{i j}\right)_{j} w_{x_{\ell}} w_{x_{i}}+4 \lambda \mu^{2} \alpha\left(\sum_{i, j=1}^{m} b^{i j} \varphi_{x_{i}} w_{x_{j}}\right)^{2}
\end{aligned}
$$

where

$$
\begin{aligned}
B= & {\left[2 \lambda^{3} \mu^{4} \alpha^{3}\left(\sum_{i, j=1}^{m} b^{i j} \varphi_{x_{i}} \varphi_{x_{j}}\right)^{2}+2 \lambda^{3} \mu^{3} \alpha^{3} \sum_{i, j=1}^{m} \sum_{k, \ell=1}^{m}\left(b^{i j} \varphi_{x_{j}} b^{k \ell} \varphi_{x_{k}} \varphi_{x_{\ell}}\right)_{x_{i}}\right.} \\
& \left.\quad-4 \lambda^{2} \mu^{2} \alpha^{2}\left(\sum_{i, j=1}^{m} b^{i j} \varphi_{x_{i} x_{j}}\right)^{2}-4 \lambda^{2} \mu^{4} \alpha^{2}\left(\sum_{i, j=1}^{m} b^{i j} \varphi_{x_{i}} \varphi_{x_{j}}\right)^{2}\right] \\
= & 2 \lambda^{3} \mu^{4} \alpha^{3}\left(\sum_{i, j=1}^{m} b^{i j} \varphi_{x_{i}} \varphi_{x_{j}}\right)^{2}-\lambda^{3} \alpha^{3} O\left(\mu^{3}\right)-\lambda^{2} \alpha^{2} O\left(\mu^{4}\right), \\
c^{i j}= & \sum_{k, \ell=1}^{m}\left[2 \lambda \mu^{2} \alpha b^{k \ell} \varphi_{x_{k}} \varphi_{x_{\ell}} b^{i j}-2 \lambda \mu \alpha b^{k \ell} \varphi_{x_{k}} b_{x_{\ell}}^{i j}-2 \lambda \mu \alpha\left(b^{k \ell} \varphi_{x_{k}}\right)_{x_{\ell}} b^{i j}\right] \\
= & \sum_{k, \ell=1}^{m} 2 \lambda \mu^{2} \alpha b^{k \ell} \varphi_{x_{k}} \varphi_{x_{\ell}} b^{i j}-\lambda \alpha O(\mu) .
\end{aligned}
$$


Proof of Lemma 2.1. We borrow some idea from [4]. First, noticing that we put only partial boundary condition on equation (2.3), we transform equation (2.3) to an equation with full boundary condition. Let

$$
b=\frac{T}{2}-\gamma, \quad b_{0}=\frac{T-T^{\prime}-\gamma}{2} .
$$

Some straightforward calculation shows that

$$
\frac{T}{2}-T^{\prime}<b_{0}<b<\frac{T}{2}
$$

We introduce $\phi(t) \in C_{0}^{\infty}\left(\frac{T}{2}-b, \frac{T}{2}+b\right)$ which enjoys the following properties

$$
\begin{cases}0 \leq \phi(t) \leq 1, & \left|t-\frac{T}{2}\right| \leq b \\ \phi(t)=1, & \left|t-\frac{T}{2}\right| \leq b_{0} .\end{cases}
$$

Let $u^{1}=\phi u$, then $u^{1}$, according to (2.3), verifies

$$
\begin{cases}u_{t t}^{1}+\sum_{i, j=1}^{n}\left(a^{i j} u_{x_{i}}^{1}\right)_{x_{j}}=\phi_{t t} u+2 \phi_{t} u_{t}, & \text { in } Q, \\ \left.u^{1}\right|_{\Gamma_{1}}=0, \int_{\Gamma_{0}} \sum_{i, j=1}^{n} a^{i j} u_{x_{i}}^{1} \nu_{j} d \Gamma=0, & \\ \left.u_{1}\right|_{\Gamma_{0}}=c \phi(t)(c \text { is unknown }), & \text { in }(\Omega \times\{0\}) \cup(\Omega \times\{T\}) . \\ u^{1}=0, & \end{cases}
$$

In what follows, we apply Lemma 4.2 to equation (4.7) with

$$
m=n+1, x_{n+1}=t,\left(b^{i j}\right)_{1 \leq i, j \leq n+1}=\left(\begin{array}{cc}
\mathcal{M} & 0 \\
0 & 1
\end{array}\right),
$$

where $\mathcal{M}=\left(a^{i j}\right)_{1 \leq i, j \leq n}, v$ replaced by $u^{1}$, and the weight function $\theta$ given in (4.1) and $w=\theta u^{1}$.

From [9], we know that there exists a $\psi \in C^{2}(\bar{\Omega})$ which enjoys the following properties:

$$
\begin{cases}\psi>0 & \text { in } \Omega \\ \psi=0 & \text { on } \Gamma \\ |\nabla \psi|>0 & \text { in } \Omega \backslash \omega_{0} \\ \frac{\partial \psi}{\partial \nu}=-\left[\sum_{i, j=1}^{n} a_{i j} \nu_{i} \nu_{j}\right]^{-\frac{1}{2}} & \text { on } \Gamma_{0}\end{cases}
$$

where $\omega_{0} \subset \subset \omega$.

Let

$$
\varrho=\frac{1}{\|\psi\|_{L^{\infty}(\Omega)}} \min _{x \in \Omega \backslash \omega}|\nabla \psi(x)| .
$$


It is clear that $\varrho>0$ following the construction of $\psi$.

Without loss of generality, we assume that $T^{\prime} \leq T-T^{\prime \prime}$. Otherwise, we can reverse the time variable $t$ to $T-t$ in equation $(2.3)$.

Let

$$
\left\{\begin{array}{l}
\varphi(x, t)=\left(c_{1}-c_{2}\right) \frac{\psi(x)}{\|\psi\|_{L^{\infty}(\Omega)}}+b^{2}-\left(t-\frac{T}{2}\right)^{2}+\kappa, \\
\widetilde{\varphi}(x, t)=\left(c_{2}-c_{1}\right) \frac{\psi(x)}{\|\psi\|_{L^{\infty}(\Omega)}}+b^{2}-\left(t-\frac{T}{2}\right)^{2}+\kappa,
\end{array}\right.
$$

where $c_{1}=b^{2}-\left(\frac{T}{2}-T^{\prime}\right)^{2}, c_{2}=\frac{1}{2}\left(c_{1}+b^{2}-b_{0}^{2}\right)$ and $\kappa$ is chosen to be so large to make $\widetilde{\varphi}>0$. It is clear that $c_{1}>c_{2}$.

Let $\alpha(x, t)=e^{\mu \varphi(x, t)}, \theta=e^{\lambda \alpha}$. According to the definition of $\alpha$, it is easy to verify that

$$
\begin{cases}\alpha(\cdot, t) \geq e^{c_{1} \mu}, & \left|t-\frac{T}{2}\right| \leq \frac{T}{2}-T^{\prime} \\ \alpha(\cdot, t) \leq e^{c_{2} \mu}, & b_{0} \leq\left|t-\frac{T}{2}\right| \leq b\end{cases}
$$

According to that $\mathcal{M}$ is uniformly positive with all elements being $C^{1}$ and $\varphi \in C^{2}\left(\mathbb{R}^{n}\right)$, some simple calculation gives that

$$
4 \lambda \mu \alpha \sum_{i, j=1}^{m} \sum_{k, \ell=1}^{m}\left(b^{k \ell} \varphi_{x_{k}} b^{i j}\right)_{x_{j}} w_{x_{\ell}} w_{x_{i}} \leq C \lambda \mu \alpha\left(\sum_{i, j=1}^{n} a^{i j} w_{x_{i}} w_{x_{j}}+\left|w_{t}\right|^{2}\right),
$$

and that

$$
\begin{aligned}
& 4 \lambda \mu^{2} \sum_{i, j=1}^{m}\left[\alpha\left(\sum_{k, \ell=1}^{m} b^{k \ell} \varphi_{x_{k}} \varphi_{\ell} b^{i j}\right)\right]_{x_{j}} w_{x_{i}} w \\
\leq & C \lambda \mu^{2} \alpha\left(\sum_{i, j=1}^{n} a^{i j} w_{x_{i}} w_{x_{j}}+\left|w_{t}\right|^{2}\right)^{\frac{1}{2}}|w| \\
\leq & C\left[\lambda^{2} \mu^{2} \alpha|w|^{2}+\mu^{2} \alpha\left(\sum_{i, j=1}^{n} a^{i j} w_{x_{i}} w_{x_{j}}+\left|w_{t}\right|^{2}\right)\right] .
\end{aligned}
$$

With the help of the construction of the function $\psi$ and the property of $\left(a^{i j}\right)_{1 \leq i, j \leq n}$, one finds that

$$
\sum_{i, j=1}^{n} a^{i j} \psi_{x_{i}} \psi_{x_{j}} \geq C|\nabla \psi|^{2}>0, \quad \text { in } \Omega \backslash \omega_{0} .
$$

This together with the definition of $\varphi$ given in (4.10) and the properties given in (4.8), implies that there exists a positive number $\mu_{0}>1$ such that for all $\mu \geq \mu_{0}$ there corresponds a positive number 
$\lambda_{0}>1$ so that

$$
\left\{\begin{aligned}
\sum_{i, j=1}^{m} c^{i j} w_{x_{i}} w_{x_{j}}-C & \left(\lambda \mu \alpha+\mu^{2}\right)\left(\sum_{i, j=1}^{n} a^{i j} w_{x_{i}} w_{x_{j}}+\left|w_{t}\right|^{2}\right) \\
& \geq \varrho^{2} \lambda \mu^{2} \alpha\left(\sum_{i, j=1}^{n} a^{i j} w_{x_{i}} w_{x_{j}}+\left|w_{t}\right|^{2}\right), \\
B w^{2}-C \lambda^{2} \mu^{4} \alpha w^{2} & \geq \varrho^{4} \lambda^{3} \mu^{4} \alpha^{3} w^{2},
\end{aligned}\right.
$$

for $\lambda \geq \lambda_{0}$ and $(x, t) \in \overline{\Omega \times(2-b, 2+b) \backslash \omega \times\left(2-b_{0}, 2+b_{0}\right)}$.

Now integrating the point-wise estimate (4.2) over $Q$, together with (4.14), we have that

$$
\begin{gathered}
\lambda \mu^{2} \int_{Q} \alpha\left(\sum_{i, j=1}^{n} a^{i j} w_{x_{i}} w_{x_{j}}+\left|w_{t}\right|^{2}\right) d x d t+\lambda^{3} \mu^{4} \int_{Q} \alpha^{3} w^{2} d x d t \\
\leq C \\
\leq \int_{Q} \theta^{2}\left|u_{t t}^{1}+\sum_{i, j=1}^{n} a^{i j} u_{x_{i} x_{j}}^{1}\right|^{2} d x d t+\lambda^{3} \mu^{4} \int_{0}^{T} \int_{\omega_{0}} \alpha^{3} w^{2} d x d t \\
\left.+\lambda \mu^{2} \int_{0}^{T} \int_{\omega_{0}} \alpha\left(\sum_{i, j=1}^{n} a^{i j} w_{x_{i}} w_{x_{j}}+\left|w_{t}\right|^{2}\right) d x d t+\int_{Q} D d x d t\right\},
\end{gathered}
$$

where

$$
\begin{aligned}
D=2 \lambda \mu \alpha \sum_{i, j=1}^{m} & {\left[2 \mu \sum_{k, \ell=1}^{m} b^{k \ell} \varphi_{x_{k}} \varphi_{x_{\ell}} b^{i j} w_{x_{i}} w+\lambda^{2} \mu^{2} \alpha^{2} \sum_{k, \ell=1}^{m} b^{k \ell} \varphi_{x_{k}} \varphi_{x_{\ell}} b^{i j} \varphi_{x_{i}} w^{2}\right.} \\
& \left.+2 \sum_{k, \ell=1}^{m} b^{k \ell} \varphi_{x_{k}} w_{x_{\ell}} b^{i j} w_{x_{i}}-\sum_{k, \ell}^{m} b^{k \ell} w_{x_{k}} w_{x_{\ell}} b^{i j} \varphi_{x_{i}}\right]_{x_{j}} .
\end{aligned}
$$

It is clear that $\int_{Q} D d x d t$ represents the boundary integral with sign not determined. Next, we will deal with this tiresome term with the appropriate choice of function $\psi$ enjoying property (4.8) and the construction of $\varphi$ given in (4.10). Denote by $V_{i}, i=1,2,3,4$ the integrals combining $\int_{Q} D d x d t$ in order. First, we have that

$$
\begin{aligned}
V_{1}= & \int_{\Sigma} 4 \lambda \mu^{2} \alpha\left(\sum_{k, \ell=1}^{n} a^{k \ell} \varphi_{x_{k}} \varphi_{x_{\ell}}+\varphi_{t}^{2}\right) \sum_{i, j=1}^{n} a^{i j} w w_{x_{i}} \nu_{j} d \Sigma \\
=\int_{\Sigma} & \left\{4 \lambda^{2} \mu^{3} \alpha^{2}\left(\sum_{k, \ell}^{n} a^{k \ell} \varphi_{x_{k}} \varphi_{x_{\ell}}+\varphi_{t}^{2}\right) \sum_{i, j=1}^{n} a^{i j} \varphi_{x_{i}} \nu_{j} w^{2}\right. \\
& \left.\quad+4 \lambda \mu^{2} \alpha \theta\left(\sum_{k, \ell=1}^{n} a^{k \ell} \varphi_{x_{k}} \varphi_{x_{\ell}}+\varphi_{t}^{2}\right) \sum_{i, j=1}^{n} a^{i j} u_{x_{i}}^{1} \nu_{j} w\right\} d \Sigma \\
= & V_{11}+V_{12} .
\end{aligned}
$$

Noticing that $\varphi_{x_{i}}=\frac{c_{1}-c_{2}}{\|\psi\|_{L^{\infty}(\Omega)}} \psi_{x_{i}}$, this together with the property of $\psi$ shows that $\sum_{i, j=1}^{n} a^{i j} \varphi_{x_{i}} \nu_{j} \leq$ 0. Also noticing that the other factors of the integrand in $V_{11}$ are all positive, then $V_{11} \leq 0$. It is 
straightforward that

$$
\sum_{k, \ell=1}^{n} a^{k \ell} \varphi_{x_{k}} \varphi_{x_{\ell}}=\left(\frac{c_{1}-c_{2}}{\|\psi\|_{L^{\infty}(\Omega)}}\right)^{2} \sum_{k, \ell=1}^{n} a^{k \ell} \psi_{x_{k}} \psi_{\ell}=\left(\frac{c_{1}-c_{2}}{\|\psi\|_{L^{\infty}(\Omega)}}\right)^{2}\left|\frac{\partial \psi}{\partial \nu}\right|^{2} \sum_{k, \ell=1}^{n} a^{k \ell} \nu_{k} \nu_{\ell}
$$

holds constant according to the property of $\psi$ and $u_{x_{i}}^{1}=\phi(t) u_{x_{i}}$. This together with that all other factors of the integrand in $V_{12}$ depending on the variable $t=x_{n+1}$ makes us conclude from the boundary condition of equation $(2.3)$ that $V_{12}=0$. Then, it holds that

$$
V_{1} \leq 0
$$

With the similar argument, it follows that

$$
V_{2}=\int_{\Sigma} 2 \lambda^{3} \mu^{3} \alpha^{3}\left(\sum_{k, \ell=1}^{n} a^{k \ell} \varphi_{x_{k}} \varphi_{x_{\ell}}+\varphi_{t}^{2}\right) \sum_{i, j=1}^{n} a^{i j} \varphi_{x_{i}} \nu_{j} w^{2} d \Sigma \leq 0 .
$$

Next, we have that

$$
\begin{aligned}
V_{3}= & \int_{\Sigma} 4 \lambda \mu \alpha\left(\sum_{k, \ell=1}^{n} a^{k \ell} \varphi_{x_{k}} \varphi_{x_{\ell}}+\varphi_{t} w_{t}\right) \sum_{i, j=1}^{n} a^{i j} w_{x_{i}} \nu_{j} d \Sigma \\
= & \int_{\Sigma}\left\{4 \lambda^{3} \mu^{3} \alpha^{3}\left(\sum_{k, \ell=1}^{n} a^{k \ell} \varphi_{x_{k}} \varphi_{x_{\ell}}+\varphi_{t}^{2}\right) \sum_{i, j=1}^{n} a^{i j} \nu_{i} \varphi_{x_{j}} w^{2}\right. \\
& +4 \lambda^{2} \mu^{2} \alpha^{2} \theta \sum_{k, \ell=1}^{n} a^{k \ell} \varphi_{x_{k}} \varphi_{x_{\ell}} w \sum_{i, j=1}^{n} a^{i j} u_{x_{i}} \nu_{j}+4 \lambda^{2} \mu^{2} \alpha^{2} \varphi_{t} \theta^{2} \sum_{i, j=1}^{n} a^{i j} \varphi_{x_{i}} \nu_{j} u^{1} u_{t}^{1} \\
& \left.-2 \lambda \mu \alpha \varphi_{t t} \theta w \sum_{i, j=1}^{n} a^{i j} u_{x_{i}} \nu_{j}+4 \lambda \mu \alpha \theta^{2} \frac{\partial \varphi}{\partial \nu}\left(\sum_{i, j=1}^{n} a^{i j} u_{x_{i}} \nu_{j}\right)^{2}\right\} d \Sigma \\
= & V_{31}+V_{32}+V_{33}+V_{34}+V_{35} .
\end{aligned}
$$

Some straightforward calculation shows that

$$
\begin{aligned}
V_{4}= & \int_{\Sigma} 2 \lambda \mu \alpha \sum_{k, \ell=1}^{m} a^{k \ell} w_{x_{k}} w_{x_{\ell}} \sum_{i, j=1}^{m} a^{i j} \varphi_{x_{i}} \nu_{j} d \Sigma \\
= & \int_{\Sigma}\left\{2 \lambda^{3} \mu^{3} \alpha^{3} \sum_{k, \ell=1}^{n} a^{k \ell} \varphi_{x_{k}} \varphi_{x_{\ell}} \sum_{i, j=1}^{n} a^{i j} \varphi_{x_{i}} \nu_{j} w^{2}+4 \lambda^{2} \mu^{2} \alpha^{2} \theta \sum_{i, j=1}^{n} a^{i j} \varphi_{x_{i}} \varphi_{x_{j}} w \sum_{k, \ell=1} a^{k \ell} u_{x_{k}} \nu_{\ell}\right. \\
& \left.+2 \lambda \mu \alpha \theta^{2} \sum_{i, j=1}^{n} a^{i j} \varphi_{x_{i}} \nu_{j} \sum_{k, \ell=1} a^{k \ell} u_{x_{k}}^{1} u_{x_{\ell}}^{1}+2 \lambda \mu \alpha\left(\lambda \mu \alpha \varphi_{t} w+\theta u_{t}^{1}\right)^{2} \sum_{i, j=1}^{n} a^{i j} \varphi_{x_{i}} \nu_{j}\right\} d \Sigma \\
= & V_{41}+V_{42}+V_{43}+V_{44} .
\end{aligned}
$$

Now some tedious calculation shows that the sign of the third term of the integrand in $V_{3}$, which involves $\varphi_{t}$ and $u_{t}^{1}$, can not be determined, thus we can not determine the sign of $\int_{Q} D d x d t$. To 
get around this difficulty, we introduce $\widetilde{\alpha}(x, t)=e^{\mu \widetilde{\varphi}(x, t)}, \widetilde{\theta}=e^{\lambda \widetilde{\alpha}}$, where $\widetilde{\varphi}$ is given in (4.10), and then apply Lemma 4.1 to equation (4.7) by letting $\widetilde{w}=\widetilde{\theta} u^{1}$.

From the definition of $\varphi, \alpha, \widetilde{\varphi}$ and $\widetilde{\alpha}$, it is easy to verify that $0<\widetilde{\varphi} \leq \varphi$ and that

$$
\left.\varphi\right|_{\Sigma}=\left.\widetilde{\varphi}\right|_{\Sigma},\left.\quad \sum_{i, j=1}^{n} a^{i j} \varphi_{x_{i}} \nu_{j}\right|_{\Sigma}=-\left.\sum_{i, j=1}^{n} a^{i j} \widetilde{\varphi}_{x_{i}} \nu_{j}\right|_{\Sigma},\left.\quad \alpha\right|_{\Sigma}=\left.\widetilde{\alpha}\right|_{\Sigma},\left.\quad w\right|_{\Sigma}=\left.\widetilde{w}\right|_{\Sigma}
$$

With the same argument of getting (4.15), some straightforward calculation tells us that

$$
\begin{gathered}
\lambda \mu^{2} \int_{Q} \widetilde{\alpha}\left(\sum_{i, j=1}^{n} a^{i j} \widetilde{w}_{x_{i}} \widetilde{w}_{x_{j}}+\left|\widetilde{w}_{t}\right|^{2}\right) d x d t+\lambda^{3} \mu^{4} \int_{Q} \widetilde{\alpha}^{3} \widetilde{w}^{2} d x d t \\
\leq C\left\{\int_{Q} \theta^{2}\left|u_{t t}^{1}+\sum_{i, j=1}^{n} a^{i j} u_{x_{i} x_{j}}^{1}\right|^{2} d x d t+\lambda^{3} \mu^{4} \int_{0}^{T} \int_{\omega_{0}} \widetilde{\alpha}^{3} \widetilde{w}^{2} d x d t\right. \\
\left.\quad+\lambda \mu^{2} \int_{0}^{T} \int_{\omega_{0}} \widetilde{\alpha}\left(\sum_{i, j=1}^{n} a^{i j} \widetilde{w}_{x_{i}} \widetilde{w}_{x_{j}}+\left|\widetilde{w}_{t}\right|^{2}\right) d x d t+\int_{Q} \widetilde{D} d x d t\right\}
\end{gathered}
$$

where

$$
\begin{aligned}
\widetilde{D}=2 \lambda \mu \widetilde{\alpha} \sum_{i, j=1}^{m}[ & 2 \mu \sum_{k, \ell=1}^{m} b^{k \ell} \widetilde{\varphi}_{x_{k}} \widetilde{\varphi}_{x_{\ell}} b^{i j} \widetilde{w}_{x_{i}} \widetilde{w}+\lambda^{2} \mu^{2} \widetilde{\alpha}^{2} \sum_{k, \ell=1}^{m} b^{k \ell} \widetilde{\varphi}_{x_{k}} \widetilde{\varphi}_{x_{\ell}} b^{i j} \widetilde{\varphi}_{x_{i}} \widetilde{w}^{2} \\
& \left.+2 \sum_{k, \ell=1}^{m} b^{k \ell} \widetilde{\varphi}_{x_{k}} \widetilde{w}_{x_{\ell}} b^{i j} \widetilde{w}_{x_{i}}-\sum_{k, \ell}^{m} b^{k \ell} \widetilde{w}_{x_{k}} \widetilde{w}_{x_{\ell}} b^{i j} \widetilde{\varphi}_{x_{i}}\right]_{x_{j}} .
\end{aligned}
$$

Similarly, $\int_{Q} \widetilde{D} d x d t$ can be transformed into a boundary integral according to Gaussian Divergence theorem. We use $\widetilde{V}_{i}, i=1,2,3,4$ to denote the integrals combining $\int_{Q} \widetilde{D} d x d t$ in their natural order as in (4.24). With the similar argument applied to $V_{i}, i=1,2,3,4$, it follows that

$$
\begin{aligned}
\widetilde{V}_{1}= & \int_{\Sigma} 4 \lambda \mu^{2} \widetilde{\alpha}\left(\sum_{k, \ell=1}^{n} a^{k \ell} \widetilde{\varphi}_{x_{k}} \widetilde{\varphi}_{x_{\ell}}+\widetilde{\varphi}_{t}^{2}\right) \sum_{i, j=1}^{n} a^{i j} \widetilde{w} \widetilde{w}_{x_{i}} \nu_{j} d \Sigma \\
= & \int_{\Sigma}\left\{4 \lambda^{2} \mu^{3} \widetilde{\alpha}^{2}\left(\sum_{k, \ell}^{n} a^{k \ell} \widetilde{\varphi}_{x_{k}} \widetilde{\varphi}_{x_{\ell}}+\widetilde{\varphi}_{t}^{2}\right) \sum_{i, j=1}^{n} a^{i j} \widetilde{\varphi}_{x_{i}} \nu_{j} \widetilde{w}^{2}\right. \\
& \left.\quad+4 \lambda \mu^{2} \widetilde{\alpha}\left(\sum_{k, \ell=1}^{n} a^{k \ell} \widetilde{\varphi}_{x_{k}} \widetilde{\varphi}_{x_{\ell}}+\widetilde{\varphi}_{t}^{2}\right) \sum_{i, j=1}^{n} a^{i j} u_{x_{i}}^{1} \nu_{j} \theta \widetilde{w}\right\} d \Sigma \\
= & \widetilde{V}_{11}+\widetilde{V}_{12} .
\end{aligned}
$$

Noticing that $\widetilde{\varphi}_{x_{i}}=\frac{c_{1}-c_{2}}{\|\psi\|_{L^{\infty}(\Omega)}} \psi_{x_{i}}$, then from the construction of $\psi$, we find that $\sum_{i, j=1}^{n} a^{i j} \widetilde{\varphi}_{x_{i}} \nu_{j}>0$. Also noticing that the other factors of the integrand in $V_{11}$ are all positive, we conclude then that $V_{11} \leq 0$. Some simple calculation shows that

$$
\sum_{k, \ell=1}^{n} a^{k \ell} \widetilde{\varphi}_{x_{k}} \widetilde{\varphi}_{x_{\ell}}=\left(\frac{c_{1}-c_{2}}{\|\psi\|_{L^{\infty}(\Omega)}}\right)^{2} \sum_{k, \ell=1}^{n} a^{k \ell} \psi_{x_{k}} \psi_{\ell}=\left(\frac{c_{1}-c_{2}}{\|\psi\|_{L^{\infty}(\Omega)}}\right)^{2}\left|\frac{\partial \psi}{\partial \nu}\right|^{2} \sum_{k, \ell=1}^{n} a^{k \ell} \nu_{k} \nu_{\ell}
$$


is constant according to the property of weight function $\psi$ and $u_{x_{i}}^{1}=\phi(t) u_{x_{i}}$. This together with that all other factors of the integrand in $V_{12}$ depends on the variable $t=x_{n+1}$, then we conclude from the boundary condition of equation (2.3) that $\widetilde{V}_{12}=0$. As a result, we conclude that

$$
\widetilde{V}_{1} \geq 0 \text {. }
$$

Using the similar argument, we have that

$$
\widetilde{V}_{2}=\int_{\Sigma} 2 \lambda^{3} \mu^{3} \widetilde{\alpha}^{3}\left(\sum_{k, \ell=1}^{n} a^{k \ell} \widetilde{\varphi}_{x_{k}} \widetilde{\varphi}_{x_{\ell}}+\widetilde{\varphi}_{t}^{2}\right) \sum_{i, j=1}^{n} a^{i j} \widetilde{\varphi}_{x_{i}} \nu_{j} \widetilde{w}^{2} d \Sigma \geq 0 .
$$

Next, we have that

$$
\begin{aligned}
\widetilde{V}_{3}= & \int_{\Sigma} 4 \lambda \mu \widetilde{\alpha}\left(\sum_{k, \ell=1}^{n} a^{k \ell} \widetilde{\varphi}_{x_{k}} \widetilde{\varphi}_{x_{\ell}}+\widetilde{\varphi}_{t} \widetilde{w}_{t}\right) \sum_{i, j=1}^{n} a^{i j} \widetilde{w}_{x_{i}} \nu_{j} d \Sigma \\
= & \int_{\Sigma}\left\{4 \lambda^{3} \mu^{3} \widetilde{\alpha}^{3}\left(\sum_{k, \ell=1}^{n} a^{k \ell} \widetilde{\varphi}_{x_{k}} \widetilde{\varphi}_{x_{\ell}}+\widetilde{\varphi}_{t}^{2}\right) \sum_{i, j=1}^{n} a^{i j} \nu_{i} \widetilde{\varphi}_{x_{j}} \widetilde{w}^{2}\right. \\
& +4 \lambda^{2} \mu^{2} \widetilde{\alpha}^{2} \widetilde{\theta} \sum_{k, \ell=1}^{n} a^{k \ell} \widetilde{\varphi}_{x_{k}} \widetilde{\varphi}_{x_{\ell}} \widetilde{w} \sum_{i, j=1}^{n} a^{i j} u_{x_{i}} \nu_{j}+4 \lambda^{2} \mu^{2} \widetilde{\alpha}^{2} \widetilde{\varphi}_{t} \widetilde{\theta}^{2} \sum_{i, j=1}^{n} a^{i j} \widetilde{\varphi}_{x_{i}} \nu_{j} u^{1} u_{t}^{1} \\
& \left.-2 \lambda \mu \widetilde{\alpha} \widetilde{\varphi} t \tilde{\theta} \widetilde{w} \sum_{i, j=1}^{n} a^{i j} u_{x_{i}} \nu_{j}+4 \lambda \mu \widetilde{\alpha} \widetilde{\theta}^{2} \frac{\partial \widetilde{\varphi}}{\partial \nu}\left(\sum_{i, j=1}^{n} a^{i j} u_{x_{i}} \nu_{j}\right)^{2}\right\} d \Sigma \\
= & \widetilde{V}_{31}+\widetilde{V}_{32}+\widetilde{V}_{33}+\widetilde{V}_{34}+\widetilde{V}_{35} .
\end{aligned}
$$

Some straightforward calculation shows that

$$
\begin{aligned}
\widetilde{V}_{4}= & \int_{\Sigma} 2 \lambda \mu \widetilde{\alpha} \sum_{k, \ell=1}^{m} a^{k \ell} \widetilde{w}_{x_{k}} \widetilde{w}_{x_{\ell}} \sum_{i, j=1}^{m} a^{i j} \widetilde{\varphi}_{x_{i}} \nu_{j} d \Sigma \\
= & \int_{\Sigma}\left\{2 \lambda^{3} \mu^{3} \widetilde{\alpha}^{3} \sum_{k, \ell=1}^{n} a^{k \ell} \widetilde{\varphi}_{x_{k}} \widetilde{\varphi}_{x_{\ell}} \sum_{i, j=1}^{n} a^{i j} \widetilde{\varphi}_{x_{i}} \nu_{j} \widetilde{w}^{2}+4 \lambda^{2} \mu^{2} \widetilde{\alpha}^{2} \widetilde{\theta} \sum_{i, j=1}^{n} a^{i j} \widetilde{\varphi}_{x_{i}} \widetilde{\varphi}_{x_{j}} \widetilde{w} \sum_{k, \ell=1} a^{k \ell} u_{x_{k}} \nu_{\ell}\right. \\
& \left.+2 \lambda \mu \widetilde{\alpha} \widetilde{\theta}^{2} \sum_{i, j=1}^{n} a^{i j} \widetilde{\varphi}_{x_{i}} \nu_{j} \sum_{k, \ell=1} a^{k \ell} u_{x_{k}}^{1} u_{x_{\ell}}^{1}+2 \lambda \mu \widetilde{\alpha}\left(\lambda \mu \widetilde{\alpha} \widetilde{\varphi}_{t} \widetilde{w}+\widetilde{\theta} u_{t}^{1}\right)^{2} \sum_{i, j=1}^{n} a^{i j} \widetilde{\varphi}_{x_{i}} \nu_{j}\right\} d \Sigma \\
= & \widetilde{V}_{41}+\widetilde{V}_{42}+\widetilde{V}_{43}+\widetilde{V}_{44} .
\end{aligned}
$$

According to (4.8), (4.22) and the boundary integral condition with respect to $u$, which solves equation (2.3), comparing $V_{1}$ with $\widetilde{V}_{1}$, it follows $V_{11}=-\widetilde{V}_{11}, V_{12}=\widetilde{V}_{12}=0$. Similarly, we find $V_{2}=-\widetilde{V}_{2}$ and $V_{31}=-\widetilde{V}_{31}, V_{32}=\widetilde{V}_{32}=0, V_{33}=-\widetilde{V}_{33}, V_{34}=-\widetilde{V}_{34}, V_{35}=-\widetilde{V}_{35}$ and $V_{41}=$ $-\widetilde{V}_{41}, V_{42}=\widetilde{V}_{42}=0, V_{43}=-\widetilde{V}_{43}, V_{44}=-\widetilde{V}_{44}$. As a result, it follows that

$$
\int_{Q} D d x d t+\int_{Q} \widetilde{D} d x d t=0
$$


Adding (4.15) to (4.23) and applying (4.30), it follows that

$$
\begin{gathered}
\lambda \mu^{2} \int_{Q}\left[\alpha\left(\sum_{i, j=1}^{n} a^{i j} w_{x_{i}} w_{x_{j}}+\left|w_{t}\right|^{2}\right)+\widetilde{\alpha}\left(\sum_{i, j=1}^{n} a^{i j} \widetilde{w}_{x_{i}} \widetilde{w}_{x_{j}}+\left|\widetilde{w}_{t}\right|^{2}\right)\right] d x d t \\
\quad+\lambda^{3} \mu^{4} \int_{Q}\left(\alpha^{3} w^{2}+\widetilde{\alpha}^{3} \widetilde{w}^{2}\right) d x d t \\
\leq C\left\{\int_{Q}\left(\theta^{2}\left|u_{t t}^{1}+\sum_{i, j=1}^{n} a^{i j} u_{x_{i} x_{j}}^{1}\right|^{2}+\widetilde{\theta}^{2}\left|u_{t t}^{1}+\sum_{i, j=1}^{n} a^{i j} u_{x_{i} x_{j}}^{1}\right|^{2}\right) d x d t\right. \\
+\lambda^{3} \mu^{4} \int_{0}^{T} \int_{\omega_{0}}\left(\alpha^{3} w^{2}+\widetilde{\alpha}^{3} \widetilde{w}^{2}\right) d x d t \\
\left.+\lambda \mu^{2} \int_{0}^{T} \int_{\omega_{0}} \alpha\left(\sum_{i, j=1}^{n} a^{i j} w_{x_{i}} w_{x_{j}}+\left|w_{t}\right|^{2}\right) d x d t\right\} .
\end{gathered}
$$

Up to now, the inequality (4.31) we got involves $w$ and $\widetilde{w}$, which is not expected for our purpose. We in the following recover $w$ and $\widetilde{w}$ to $u^{1}$. Recalling that $w=\theta u^{1}$ and $\widetilde{w}=\widetilde{\theta} u^{1}$, some straightforward calculation gives that

$$
\begin{aligned}
& \frac{1}{C} \theta^{2}\left(\sum_{i, j=1}^{n} a^{i j} u_{x_{i}}^{1} u_{x_{j}}^{1}+\left|u_{t}^{1}\right|^{2}+\lambda^{2} \mu^{2} \alpha^{2}\left|u^{1}\right|^{2}\right) \\
\leq & \sum_{i, j=1}^{n} a^{i j} w_{x_{i}} w_{x_{j}}+\left|w_{t}\right|^{2}+\lambda^{2} \mu^{2} \alpha^{2} w^{2} \\
\leq & C \theta^{2}\left(\sum_{i, j=1}^{n} a^{i j} u_{x_{i}}^{1} u_{x_{j}}^{1}+\left|u_{t}^{1}\right|^{2}+\lambda^{2} \mu^{2} \alpha^{2}\left|u^{1}\right|^{2}\right) .
\end{aligned}
$$

Similarly, it follows that

$$
\begin{aligned}
& \frac{1}{C} \widetilde{\theta}^{2}\left(\sum_{i, j=1}^{n} a^{i j} u_{x_{i}}^{1} u_{x_{j}}^{1}+\left|u_{t}^{1}\right|^{2}+\lambda^{2} \mu^{2} \widetilde{\alpha}^{2}\left|u^{1}\right|^{2}\right) \\
\leq & \sum_{i, j=1}^{n} a^{i j} \widetilde{w}_{x_{i}} \widetilde{w}_{x_{j}}+\left|\widetilde{w}_{t}\right|^{2}+\lambda^{2} \mu^{2} \widetilde{\alpha}^{2} \widetilde{w}^{2} \\
\leq & C \widetilde{\theta}^{2}\left(\sum_{i, j=1}^{n} a^{i j} u_{x_{i}}^{1} u_{x_{j}}^{1}+\left|u_{t}^{1}\right|^{2}+\lambda^{2} \mu^{2} \widetilde{\alpha}^{2}\left|u^{1}\right|^{2}\right) .
\end{aligned}
$$

We also need to get ride of $\widetilde{\alpha}$ and $\widetilde{\theta}$ appeared in (4.31). By means of the definition of $\alpha, \widetilde{\alpha}, \theta, \widetilde{\theta}$, 
it is easy to verified that $\alpha \geq \widetilde{\alpha} \geq 1$ and $\theta \geq \widetilde{\theta}>1$, which in turn gives that

$$
\left\{\begin{array}{l}
\widetilde{\alpha} \widetilde{\theta}^{2}\left(\sum_{i, j=1}^{n} a^{i j} u_{x_{i}}^{1} u_{x_{j}}^{1}+\left|u_{t}^{1}\right|^{2}\right) \leq \alpha \theta^{2}\left(\sum_{i, j=1}^{n} a^{i j} u_{x_{i}}^{1} u_{x_{j}}^{1}+\left|u_{t}^{1}\right|^{2}\right), \\
\widetilde{\theta}^{2}\left|\sum_{i, j=1}^{n} a^{i j} u_{x_{i} x_{j}}^{1}\right|^{2} \leq \theta^{2}\left|\sum_{i, j=1}^{n} a^{i j} u_{x_{i} x_{j}}^{1}\right|^{2}, \\
\widetilde{\alpha}^{3} \widetilde{\theta}^{2}\left|u^{1}\right|^{2} \leq \alpha^{3} \theta^{2}\left|u^{1}\right|^{2} .
\end{array}\right.
$$

By means of (4.31)- (4.34), it follows that

$$
\begin{gathered}
\lambda \mu^{2} \int_{Q} \alpha \theta^{2}\left(\sum_{i, j=1}^{n} a^{i j} u_{x_{i}}^{1} u_{x_{j}}^{1}+\left|u_{t}^{1}\right|^{2}\right)+\lambda^{3} \mu^{4} \int_{Q} \alpha^{3} \theta^{2}\left|u^{1}\right|^{2} d x d t \\
\leq C\left\{\int_{Q} \theta^{2}\left|u_{t t}^{1}+\sum_{i, j=1}^{n} a^{i j} u_{x_{i} x_{j}}^{1}\right|^{2} d x d t+\lambda^{3} \mu^{4} \int_{0}^{T} \int_{\omega_{0}} \alpha^{3} \theta^{2}\left|u^{1}\right|^{2} d x d t\right. \\
\left.+\lambda \mu^{2} \int_{0}^{T} \int_{\omega_{0}} \alpha \theta^{2}\left(\sum_{i, j=1}^{n} a^{i j} u_{x_{i}}^{1} u_{x_{j}}^{1}+\left|u_{t}^{1}\right|^{2}\right) d x d t\right\} .
\end{gathered}
$$

Noticing that $u^{1}$ solves equation (4.7), it is easy for one to verify that

$$
\begin{aligned}
\left|\sum_{i, j=1}^{n} a^{i j} u^{1} x_{i} x_{j}+u_{t t}^{1}\right|^{2} & \leq 2\left|\phi_{t t} u+2 \phi_{t} u_{t}\right|^{2}+2\left|\sum_{i, j=1}^{n} a_{x_{j}}^{i j} u_{x_{i}}^{1}\right|^{2} \\
& \leq 2\left|\phi_{t t} u+2 \phi_{t} u_{t}\right|^{2}+C\left|\nabla u^{1}\right|^{2}
\end{aligned}
$$

Based on that $\mathcal{M}$ is uniformly positive, it follows that

$$
\sum_{i, j=1}^{n} a^{i j} u_{x_{i}}^{1} u_{x_{j}}^{1}+\left|u_{t}^{1}\right|^{2} \geq C\left(\left|\nabla u^{1}\right|^{2}+\left|u_{t}^{1}\right|^{2}\right)
$$

Take $\chi \in C_{0}^{\infty}(\omega)$ to be such that $0 \leq \chi \leq 1$ and $\chi=1$ in $\omega_{0}$. Multiplying equation (4.7) from both sides by $\chi \theta^{2} \alpha u^{1}$ and then integrating by parts, we arrive at

$$
\begin{aligned}
& \int_{0}^{T} \int_{\omega_{0}} \alpha \theta^{2}\left(\sum_{i, j=1}^{n} a^{i j} u_{x_{i}}^{1} u_{x_{j}}^{1}+\left|u_{t}^{1}\right|^{2}\right) d x d t \\
\leq & C\left[\lambda \mu^{2} \int_{0}^{T} \int_{\omega} \alpha^{2} \theta^{2}\left|u^{1}\right|^{2} d x d t+\int_{Q} \theta^{2}\left|\phi_{t t} u+2 \phi_{t} u_{t}\right|^{2} d x d t\right] .
\end{aligned}
$$

By means of (4.35)- (4.38), it follows that

$$
\begin{aligned}
& \lambda \mu^{2} \int_{Q} \alpha \theta^{2}\left(\left|\nabla u^{1}\right|^{2}+\left|u_{t}^{1}\right|^{2}\right) d x d t-C \int_{Q} \theta^{2}\left|\nabla u^{1}\right|^{2} d x d t+\lambda^{3} \mu^{4} \int_{Q} \alpha^{3} \theta^{2}\left|u^{1}\right|^{2} d x d t \\
\leq & C\left\{\int_{Q} \theta^{2}\left|\phi_{t t} u+2 \phi_{t} u_{t}\right|^{2} d x d t+\lambda^{3} \mu^{4} \int_{0}^{T} \int_{\omega} \alpha^{3} \theta^{2}\left|u^{1}\right|^{2} d x d t\right\} .
\end{aligned}
$$


It is easy to see that there must be some $\mu_{1} \geq \mu_{0}$, such that for any $\mu \geq \mu_{1}$, we have the following inequality

$$
\begin{aligned}
& \lambda^{3} \mu^{4} \int_{Q} \alpha^{3} \theta^{2}\left|u^{1}\right|^{2} d x d t \\
\leq & C\left\{\int_{Q} \theta^{2}\left|\phi_{t t} u+2 \phi_{t} u_{t}\right|^{2} d x d t+\lambda^{3} \mu^{4} \int_{0}^{T} \int_{\omega} \alpha^{3} \theta^{2}\left|u^{1}\right|^{2} d x d t\right\} .
\end{aligned}
$$

We need do some estimates on each term in both sides of (4.40). With the help of (4.11) and (4.6), one finds that the term in the left hand side of (4.40) is given as follows:

$$
\lambda^{3} \mu^{4} \int_{Q} \alpha^{3} \theta^{2}\left|u^{1}\right|^{2} d x d t \geq \lambda^{3} \mu^{4} e^{3 \mu\left(c_{1}+\kappa\right)} e^{2 \lambda e^{\mu\left(c_{1}+\kappa\right)}} \int_{T^{\prime}}^{T^{\prime \prime}} \int_{\Omega} u^{2} d x d t .
$$

Similarly, we have the estimates for the two terms in the right hand side of (4.40) respectively as follows:

$$
\begin{aligned}
& \int_{Q} \theta^{2}\left|\phi_{t t} u+2 \phi_{t} u_{t}\right|^{2} d x d t \\
= & \int_{\frac{T}{2}-b}^{\frac{T}{2}+b} \int_{\Omega} \theta^{2}\left|\phi_{t t} u+2 \phi_{t} u_{t}\right|^{2} d x d t \\
\leq & C e^{2 \lambda e^{\mu\left(c_{2}+\kappa\right)}}\left\{\int_{\frac{T}{2}-b}^{\frac{T}{2}-b_{0}} \int_{\Omega}\left(u^{2}+u_{t}^{2}\right) d x d t+\int_{\frac{T}{2}+b_{0}}^{\frac{T}{2}+b} \int_{\Omega}\left(u^{2}+u_{t}^{2}\right) d x d t\right\} \\
\leq & C e^{2 \lambda e^{\mu\left(c_{2}+\kappa\right)}}\|u\|_{H^{1}(Q)}^{2},
\end{aligned}
$$

and

$$
\begin{aligned}
\lambda^{3} \mu^{4} \int_{0}^{T} \int_{\omega} \alpha^{3} \theta^{2}\left|u^{1}\right|^{2} d x d t & =\lambda^{3} \mu^{4} \int_{\frac{T}{2}-b}^{\frac{T}{2}+b} \int_{\omega} \alpha^{3} \theta^{2}\left|u^{1}\right|^{2} d x d t \\
& \leq \lambda^{3} \mu^{4} e^{3 \mu\left(\frac{T^{2}}{4}+c_{1}-c_{2}+\kappa\right)} e^{2 \lambda e^{\mu\left(\frac{T^{2}}{4}+c_{1}-c_{2}+\kappa\right)}} \int_{\frac{T}{2}-b}^{\frac{T}{2}+b} \int_{\omega} u^{2} d x d t .
\end{aligned}
$$

Putting the estimates $(4.41)-(4.43)$ into (4.40), we conclude that

$$
\begin{aligned}
& \lambda^{3} \mu^{4} e^{3 \mu\left(c_{1}+\kappa\right)} e^{2 \lambda e^{\mu\left(c_{1}+\kappa\right)}} \int_{T^{\prime}}^{T^{\prime \prime}} \int_{\Omega} u^{2} d x d t \\
\leq & \lambda^{3} \mu^{4} e^{3 \mu\left(\frac{T^{2}}{4}+c_{1}-c_{2}+\kappa\right)} e^{2 \lambda e^{\mu\left(\frac{T^{2}}{4}+c_{1}-c_{2}+\kappa\right)}} \int_{\frac{T}{2}-b}^{\frac{T}{2}+b} \int_{\omega} u^{2} d x d t+C e^{2 \lambda e^{\mu\left(c_{2}+\kappa\right)}}\|u\|_{H^{1}(Q)}^{2} .
\end{aligned}
$$

Noticing by definition that $c_{1}>c_{2}$, it follows that $e^{2 \lambda e^{\mu\left(c_{1}+\kappa\right)}}>e^{2 \lambda e^{\mu\left(c_{2}+\kappa\right)}}$. Fixing $\mu=\mu_{1}$, letting

$$
\left\{\begin{array}{l}
\varepsilon=\frac{e^{2 \lambda e^{\mu_{1}\left(c_{2}+\kappa\right)}}}{\lambda^{3} \mu_{1}^{4} e^{3 \mu_{1}\left(c_{1}+\kappa\right)} e^{2 \lambda e^{\mu_{1}\left(c_{1}+\kappa\right)}}, \quad k=\frac{e^{\mu_{1}\left(\frac{T^{2}}{4}+c_{1}-c_{2}+\kappa\right)}-e^{\mu_{1}\left(c_{1}+\kappa\right)}}{e^{\mu_{1}\left(c_{1}+\kappa\right)}-e^{\mu_{1}\left(c_{2}+\kappa\right)}},} \\
\varepsilon_{0}=\frac{e^{2 \lambda_{0} e^{\mu_{1}\left(c_{2}+\kappa\right)}}}{\lambda_{0}^{3} \mu_{1}^{4} e^{3 \mu_{1}\left(c_{1}+\kappa\right)} e^{2 \lambda_{0} e^{\mu_{1}\left(c_{1}+\kappa\right)}}},
\end{array}\right.
$$


thus based on (4.44), one finds that for any $\varepsilon \in\left(0, \varepsilon_{0}\right]$ the following inequality holds:

$$
\|u\|_{L^{2}\left(\Omega \times\left(T^{\prime}, T^{\prime \prime}\right)\right.} \leq \varepsilon^{-k}\|u\|_{L^{2}(\omega \times(\gamma, T-\gamma))}+C \varepsilon\|u\|_{H^{1}(Q)} .
$$

Therefore, (4.45) holds for all $\varepsilon>0$. Further, if we let $\tau=\frac{1}{1+k}, \varepsilon=\left(\frac{\|u\|_{L^{2}(\omega \times(\gamma, T-\gamma))}}{\|u\|_{H^{1}(Q)}^{1-\tau}}\right)^{2 \tau},(4.45)$ in turn gives that

$$
\|u\|_{L^{2}\left(\Omega \times\left(T^{\prime}, T^{\prime \prime}\right)\right)} \leq C\|u\|_{L^{2}(\omega \times(\gamma, T-\gamma))}^{\tau}\|u\|_{H^{1}(\Omega \times(0, T))}^{1-\tau}
$$

as desired. We then complete the proof of Lemma 2.1 .

Proof of Lemma 2.2. Here and thereafter, we use the symbol dist $\left((x, t), \omega_{0} \times\{0\}\right)$ to denote the distance between the point $(x, t)$ and the set $\omega_{0} \times\{0\}$. Let

$$
N(\tau)=\left\{(x, t) \mid(x, t) \in Q, \operatorname{dist}\left((x, t), \omega_{0} \times\{0\}\right)<\tau\right\} .
$$

Let $\tau_{i}(i=1,2,3)$ be such that $0<\tau_{1}<\tau_{2}<\tau_{3}$ and $N\left(\tau_{3}\right) \subset Q$ and $N\left(\tau_{3}\right) \cap(\Omega \times\{0\}) \subset$ $(\omega \times\{0\})$. We take a $C^{2}$ function $h(x, t)$ with $3<h<4$ when $(x, t) \in N\left(\tau_{1}\right)$ but $0<h<1$ when $(x, t) \in N\left(\tau_{3}\right) \backslash N\left(\tau_{2}\right)$ and $|\nabla h|>0$ in $N\left(\tau_{3}\right)$. The proof of the existence of such function can be found in [4]. But for easy reference, we give it here. Let $g: \mathbb{R} \rightarrow \mathbb{R}$ be such that: $g^{\prime}<0$; $3<g(s)<4$ for $s \in\left(0, \tau_{1}^{2}\right) ; 0<g(s)<1$ for $s \in\left(\tau_{2}^{2}, \tau_{3}^{2}\right)$. Let $h(x, t)=g\left(\operatorname{dist}^{2}\left((x, t), \omega_{0} \times\{0\}\right)\right)$, which is expected.

Now we take $\chi \in C^{\infty}\left(N\left(\tau_{3}\right)\right)$ with the properties $0 \leq \chi \leq 1$ and $\chi=1$ in $N\left(\tau_{2}\right)$ and vanishes in the intersection of $N\left(\tau_{3}\right)$ and a neighborhood, which is very small, of $\partial N\left(\tau_{3}\right) \backslash(\omega \times\{0\})$.

For any $u \in H^{2}(Q)$, which solves equation (2.3), we let $\bar{u}=\chi u$, then $\bar{u}$ solves

$$
\begin{cases}\bar{u}_{t t}+\sum_{i, j=1}^{n}\left(a^{i j} \bar{u}_{x_{i}}\right)_{x_{j}}= & \chi_{t t} u+2 \chi_{t} u_{t}+\sum_{i, j=1}^{n} a^{i j} \chi_{x_{i} x_{j}} u \\ & +2 \sum_{i, j=1}^{n} a^{i j} \chi_{x_{i}} u_{x_{j}}+\sum_{i, j=1}^{n} a_{x_{j}}^{i j} \chi_{x_{j}} u, \quad(x, t) \in N\left(\tau_{3}\right), \\ |\nabla \bar{u}|=\bar{u}=0, & (x, t) \in \partial N\left(\tau_{3}\right) \backslash(\omega \times\{0\}) .\end{cases}
$$

Like the procedure for proving Lemma 2.1, we also let $m=n+1, x_{n+1}=t$ and $\left(b^{i j}\right)_{1 \leq i, j \leq n+1}$ be given in the same manner. To apply Lemma 4.1 to equation (4.47), we here let $\theta=e^{\lambda e^{\mu h}}$ and replace $v$ by $\bar{u}$, that is, $w=\theta \bar{u}$.

Some straightforward calculation gives that

$$
\begin{aligned}
& \lambda \mu^{2} \int_{N\left(\tau_{3}\right)} \alpha\left(\sum_{i, j=1}^{n} a^{i j} w_{x_{i}} w_{x_{j}}+w_{t}^{2}\right) d x d t+\lambda^{3} \mu^{4} \int_{N\left(\tau_{3}\right)} \alpha^{3} w^{2} d x d t \\
\leq & C\left\{\int_{N\left(\tau_{3}\right)} \theta^{2}\left|\bar{u}_{t t}+\sum_{i, j=1}^{n} a^{i j} \bar{u}_{x_{i} x_{j}}\right|^{2} d x d t+\int_{N\left(\tau_{3}\right)} D_{1} d x d t\right\},
\end{aligned}
$$


where

$$
\begin{aligned}
D_{1}=2 \lambda \mu \alpha \sum_{i, j=1}^{m}[ & 2 \mu \sum_{k, \ell} b^{k \ell} h_{x_{k}} h_{x_{\ell}} b^{i j} w_{x_{k}} w_{x_{\ell}}+\lambda^{2} \mu^{2} \alpha^{2} \sum_{k, \ell=1}^{m} b^{k \ell} h_{x_{k}} h_{x_{\ell}} b^{i j} h_{x_{i}} w^{2} \\
& \left.+2 \sum_{k, \ell=1}^{m} b^{k \ell} h_{x_{k}} w_{x_{\ell}} b^{i j} w_{x_{i}}-\sum_{k, \ell=1}^{m} b^{k \ell} w_{x_{k}} w_{x_{\ell}} b^{i j} h_{x_{i}}\right]_{x_{j}} .
\end{aligned}
$$

Of course, $\int_{N\left(\tau_{3}\right)} D_{1} d x d t$ stands for the boundary integral according to Gaussian Divergence theorem, which is not welcome here. We in what follows to estimate this integral term by term so that we can transform it to some term suitable for our purpose. For simplicity, we adopt $\bar{V}_{i}, i=1,2,3,4$ to denote the integral consisting $\int_{N\left(\tau_{3}\right)} D_{1} d x d t$ in their natural order as given in (4.49). Remember the definition of $w$, it follows that

$$
\left\{\begin{array}{l}
\left.w\right|_{\partial N\left(\tau_{3}\right) \backslash(\omega \times\{0\})}=\bar{u}_{\partial N\left(\tau_{3}\right) \backslash(\omega \times\{0\})}=0, \\
\left.\nabla w\right|_{\partial N\left(\tau_{3}\right) \backslash(\omega \times\{0\})}=\nabla \bar{u}_{\partial N\left(\tau_{3}\right) \backslash(\omega \times\{0\})}=0,
\end{array}\right.
$$

which in turn inspires that

$$
\begin{aligned}
\bar{V}_{1} & =\int_{\partial N\left(\tau_{3}\right)} 4 \lambda \mu^{2} \alpha \sum_{k, \ell=1}^{m} b^{k \ell} h_{x_{k}} h_{x_{\ell}} \sum_{i, j=1}^{m} b^{i j} w_{x_{i}} \nu_{j} w d \partial N\left(\tau_{3}\right) \\
& \leq C \int_{\partial N\left(\tau_{3}\right)}\left\{\lambda \mu \alpha\left(|\nabla w|^{2}+w_{t}^{2}\right)+\lambda \mu^{3} \alpha w^{2}\right\} d N\left(\tau_{3}\right) \\
& \leq C \int_{\omega \times\{0\}}\left\{\lambda \mu \alpha\left(|\nabla w|^{2}+w_{t}^{2}\right)+\lambda \mu^{3} w^{2}\right\} d x .
\end{aligned}
$$

By the same manner, we have

$$
\begin{gathered}
\bar{V}_{2}=\int_{\partial N\left(\tau_{3}\right)} 2 \lambda^{3} \mu^{3} \alpha^{3} \sum_{k, \ell=1}^{m} b^{k \ell} h_{x_{k}} h_{x_{\ell}} \sum_{i, j=1}^{m} b^{i j} h_{x_{i}} \nu_{j} w^{2} d N\left(\tau_{3}\right) \leq C \int_{\omega \times\{0\}} \lambda^{3} \mu^{3} \alpha^{3} w^{2} d x \\
\bar{V}_{3}=\int_{\partial N\left(\tau_{3}\right)} 4 \lambda \mu \alpha \sum_{k, \ell=1}^{m} b^{k \ell} h_{x_{k}} w_{x_{\ell}} \sum_{i, j=1}^{m} b^{i j} w_{x_{i}} \nu_{j} d N\left(\tau_{3}\right) \leq C \int_{\omega \times\{0\}} \lambda \mu \alpha\left(|\nabla w|^{2}+w_{t}^{2}\right) d x
\end{gathered}
$$

and

$$
\bar{V}_{4}=\int_{\partial N\left(\tau_{3}\right)} 2 \lambda \mu \alpha \sum_{k, \ell=1}^{m} b^{k \ell} w_{x_{k}} w_{x_{\ell}} \sum_{i, j=1}^{m} b^{i j} h_{x_{i}} \nu_{j} d N\left(\tau_{3}\right) \leq C \int_{\omega \times\{0\}} \lambda \mu \alpha\left(|\nabla w|^{2}+w_{t}^{2}\right) d x .
$$

Combining $(4.51)-(4.54)$, one finds that

$$
\begin{aligned}
\int_{N\left(\tau_{3}\right)} D_{1} d x & =\bar{V}_{1}+\bar{V}_{2}+\bar{V}_{3}+\bar{V}_{4} \\
& \leq C \int_{\omega \times\{0\}}\left\{\lambda \mu \alpha\left(|\nabla w|^{2}+w_{t}^{2}\right)+\lambda^{3} \mu^{3} \alpha^{3} w^{2}\right\} d x .
\end{aligned}
$$


Returning back to (4.48), we have that

$$
\begin{aligned}
& \lambda \mu^{2} \int_{N\left(\tau_{3}\right)} \alpha\left(\sum_{i, j=1}^{n} a^{i j} w_{x_{i}} w_{x_{j}}+w_{t}^{2}\right) d x d t+\lambda^{3} \mu^{4} \int_{N\left(\tau_{3}\right)} \alpha^{3} w^{2} d x d t \\
\leq & C\left\{\int_{N\left(\tau_{3}\right)} \theta^{2}\left|\bar{u}_{t t}+\sum_{i, j=1}^{n} a^{i j} \bar{u}_{x_{i} x_{j}}\right|^{2} d x d t+\int_{\omega \times\{0\}}\left[\lambda \mu \alpha\left(|\nabla w|^{2}+w_{t}^{2}\right)+\lambda^{3} \mu^{3} \alpha^{3} w^{2}\right] d x\right\} .
\end{aligned}
$$

Next, we are to recover $\bar{u}$ from $w$. Based on $w=\theta \bar{u}$, it is easy to show that

$$
\begin{aligned}
& \frac{1}{C} \theta^{2}\left(\sum_{i, j=1}^{n} a^{i j} \bar{u}_{x_{i}} \bar{u}_{x_{j}}+\bar{u}_{t}^{2}+\lambda^{2} \mu^{2} \alpha^{2} \bar{u}^{2}\right) \\
\leq & \sum_{i, j=1}^{n} w_{x_{i}} w_{x_{j}}+w_{t}^{2}+\lambda^{2} \mu^{2} \alpha^{2} w^{2} \\
\leq & C \theta^{2}\left(\sum_{i, j=1}^{n} a^{i j} \bar{u}_{x_{i}} \bar{u}_{x_{j}}+\bar{u}_{t}^{2}+\lambda^{2} \mu^{2} \alpha^{2} \bar{u}^{2}\right) .
\end{aligned}
$$

For that $\mathcal{M}$ is uniformly positive, it gives that

$$
\sum_{i, j=1}^{n} a^{i j} \bar{u}_{x_{i}} \bar{u}_{x_{j}}+\bar{u}_{t}^{2} \geq C\left(|\nabla \bar{u}|^{2}+\bar{u}_{t}^{2}\right) .
$$

On the other hand, for $\bar{u}$ solves equation (4.47), one can show that

$$
\begin{aligned}
& \left|\sum_{i, j=1}^{n} a^{i j} \bar{u}_{x_{i} x_{j}}+\bar{u}_{t t}\right|^{2} \\
\leq & 2\left|\chi_{t t} u+2 \chi_{t} u_{t}+2 \sum_{i, j=1}^{n} a^{i j} \chi_{x_{i}} u_{x_{j}}+\sum_{i, j=1}^{n} a^{i j} \chi_{x_{i} x_{j}} u\right|^{2}+2\left|\sum_{i, j=1}^{n} a_{x_{j}}^{i j} u_{x_{i}}\right|^{2} \\
\leq & 2\left|\chi_{t t} u+2 \chi_{t} u_{t}+2 \sum_{i, j=1}^{n} a^{i j} \chi_{x_{i}} u_{x_{j}}+\sum_{i, j=1}^{n} a^{i j} \chi_{x_{i} x_{j}} u\right|^{2}+C|\nabla u|^{2} .
\end{aligned}
$$

Then (4.57) - (4.59) together with (4.56) gives that

$$
\begin{aligned}
& \lambda \mu^{2} \int_{N\left(\tau_{3}\right)}\left(|\nabla \bar{u}|^{2}+\bar{u}_{t}^{2}\right) d x d t-C \int_{N\left(\tau_{3}\right)} \theta^{2}|\nabla \bar{u}|^{2} d x d t+\lambda^{3} \mu^{4} \int_{N\left(\tau_{3}\right)} \alpha^{3} \theta^{2} \bar{u}^{2} d x d t \\
\leq & C\left\{\int_{N\left(\tau_{3}\right)} \theta^{2}\left|\chi_{t t} u+2 \chi_{t} u_{t}+2 \sum_{i, j=1}^{n} a^{i j} \chi_{x_{i}} u_{x_{j}}+\sum_{i, j=1}^{n} a^{i j} \chi_{x_{i} x_{j}} u\right|^{2}\right. \\
& \left.+\int_{\omega \times\{0\}}\left[\lambda \mu \alpha\left(|\nabla w|^{2}+w_{t}^{2}\right)+\lambda^{3} \mu^{3} \alpha^{3} w^{2}\right] d x\right\} .
\end{aligned}
$$


As a result, there must be some $\mu_{2}>0$ such that for any $\mu \geq \mu_{2}$, it holds that

$$
\begin{gathered}
\lambda \mu^{2} \int_{N\left(\tau_{3}\right)} \alpha \theta^{2}\left(|\nabla \bar{u}|^{2}+\bar{u}_{t}^{2}\right) d x d t+\lambda^{3} \mu^{4} \int_{N\left(\tau_{3}\right)} \alpha^{3} \theta^{2} \bar{u}^{2} d x d t \\
\leq C \\
\leq \int_{N\left(\tau_{3}\right)} \theta^{2}\left|\chi_{t t} u+2 \chi_{t} u_{t}+2 \sum_{i, j=1}^{n} \chi_{x_{i}} u_{x_{j}}+\sum_{i, j=1}^{n} \chi_{x_{i} x_{j}} u\right|^{2} d x d t \\
\left.+\int_{\omega \times\{0\}}\left[\lambda \mu \alpha\left(|\nabla \bar{u}|^{2}+\bar{u}_{t}^{2}\right)+\lambda^{3} \mu^{3} \theta^{2} \bar{u}^{2}\right] d x\right\} .
\end{gathered}
$$

Furthermore, noticing that $\bar{u}=u$ as $(x, t) \in N\left(\tau_{3}\right), \chi_{t}=0, x_{x_{i}}=0$ as $(x, t) \in N\left(\tau_{2}\right)$ and $\alpha>e^{3 \mu}, \theta>e^{\lambda e^{3 \mu}}$ as $(x, t) \in N\left(\tau_{1}\right)$ but $\alpha<e^{\mu}, \theta<e^{\lambda e^{\mu}}$ as $(x, t) \in N\left(\tau_{3}\right) \backslash N\left(\tau_{2}\right)$, we can conclude that the following several inequalities:

$$
\begin{aligned}
& \int_{N\left(\tau_{3}\right)} \lambda \mu^{2} \alpha \theta^{2}\left(|\nabla \bar{u}|^{2}+\bar{u}_{t}^{2}\right) d x d t \geq \lambda \mu^{2} e^{3 \mu} e^{2 \lambda e^{3 \mu}} \int_{N\left(\tau_{3}\right)}\left(|\nabla \bar{u}|^{2}+\bar{u}_{t}^{2}\right) d x d t, \\
& \int_{N\left(\tau_{3}\right)} \lambda^{3} \mu^{4} \alpha^{3} \theta^{2} \bar{u}^{2} d x d t \geq \lambda^{3} \mu^{4} e^{9 \mu} e^{2 \lambda e^{3 \mu}} \int_{N\left(\tau_{1}\right)} u^{2} d x d t \\
& \int_{N\left(\tau_{3}\right)} \theta^{2}\left|\chi_{t t} u+2 \chi_{t} u_{t}+2 \sum_{i, j=1}^{n} a^{i j} \chi_{x_{i}} u_{x_{j}}+\sum_{i, j=1}^{n} a^{i j} \chi_{x_{i} x_{j}} u\right|^{2} d x d t \\
& \leq C e^{2 \lambda e^{\mu}} \int_{N\left(\tau_{3}\right)}\left(u^{2}+|\nabla u|^{2}+u_{t}^{2}\right) d x d t, \\
& \int_{\omega \times\{0\}} \lambda^{3} \mu^{3} \alpha^{3} \theta^{2} \bar{u}^{2} d x \leq \lambda^{3} \mu^{3} e^{12 \mu} e^{2 \lambda e^{4 \mu}} \int_{\omega \times\{0\}} u^{2} d x, \\
& \int_{\omega \times\{0\}} \lambda \mu \alpha \theta^{2}\left(|\nabla \bar{u}|^{2}+\bar{u}_{t}^{2}\right) d x \leq \lambda \mu e^{4 \mu} e^{2 \lambda e^{4 \mu}} \int_{\omega \times\{0\}}\left(|\nabla u|^{2}+u_{t}^{2}\right) d x .
\end{aligned}
$$

Then (4.62) - (4.66) together with (4.61) gives that

$$
\begin{aligned}
& \lambda \mu^{2} e^{3 \mu} e^{2 \lambda e^{3 \mu}} \int_{N\left(\tau_{1}\right)}\left(|\nabla \bar{u}|^{2}+\bar{u}_{t}^{2}\right)+\lambda^{3} \mu^{4} e^{9 \mu} e^{2 \lambda e^{3 \mu}} \int_{N\left(\tau_{1}\right)} u^{2} d x d t \\
& \leq C\left\{\int_{N\left(\tau_{3}\right)}\left(u^{2}+|\nabla u|^{2}+u_{t}^{2}\right)+\lambda^{3} \mu^{3} e^{12 \mu} e^{2 \lambda e^{4 \mu}} \int_{\omega \times\{0\}} u^{2} d x\right. \\
&\left.+\lambda \mu e^{4 \mu} e^{2 \lambda e^{4 \mu}} \int_{\omega \times\{0\}}\left(|\nabla u|^{2}+u_{t}^{2}\right) d x\right\}
\end{aligned}
$$

With the similar argument for (4.45), some straightforward calculation shows that there must be some $\beta>0$ and $\varepsilon_{0}>0$ such that for any $\varepsilon \in\left(0, \varepsilon_{0}\right]$ it follows that

$$
\|u\|_{H^{1}\left(N\left(\tau_{1}\right)\right)}^{2} \leq \varepsilon^{-\beta}\left(\|u(0)\|_{L^{2}(\omega)}+\left\|u_{t}\right\|_{L^{2}(\omega)}+\|\nabla u\|_{L^{2}(\omega)}\right)+C \varepsilon\|u\|_{H^{1}(Q)},
$$


which implies (4.68) itself holding for any $\varepsilon>0$.

Next, noticing that $\tau_{1}>0$, there therefore must be some open ball $B \subset N\left(\tau_{1}\right)$. It follows from (4.68) that

$$
\|u\|_{H^{1}(B)}^{2} \leq \varepsilon^{-\beta}\left(\|u(0)\|_{L^{2}(\omega)}+\left\|u_{t}\right\|_{L^{2}(\omega)}+\|\nabla u\|_{L^{2}(\omega)}\right)+C \varepsilon\|u\|_{H^{1}(Q)} .
$$

In the above inequality, if we let

$$
\delta^{\prime}=\frac{1}{1+\beta}, \quad \varepsilon=\frac{\|u(0)\|_{L^{2}(\omega)}+\left\|u_{t}\right\|_{L^{2}(\omega)}+\|\nabla u\|_{L^{2}(\omega)}}{\|u\|_{H^{1}(Q)}},
$$

then it follows that

$$
\|u\|_{H^{1}(B)} \leq C\left(\|u(0)\|_{L^{2}(\omega)}+\left\|u_{t}\right\|_{L^{2}(\omega)}+\|\nabla u\|_{L^{2}(\omega)}\right)^{\delta^{\prime}}\|u\|_{H^{1}(Q)}^{1-\delta^{\prime}} .
$$

We now do the following assertion: for any $K \subset \subset Q$, there must be some $\delta^{\prime \prime}$ with $0<\delta^{\prime \prime}<1$ such that

$$
\|u\|_{H^{1}(K)} \leq C\|u\|_{H^{1}(B)}^{\delta^{\prime \prime}}\left\|H^{1}(Q)\right\|^{1-\delta^{\prime \prime}} .
$$

We will prove this assertion later. According to the assertion (4.71) together with (4.70), taking $K=\omega \times(\gamma, T-\gamma) \subset \subset Q$, it follows that

$$
\|u\|_{H^{1}(\omega \times(\gamma, T-\gamma))} \leq C\left(\|u(0)\|_{L^{2}(\omega)}+\left\|u_{t}\right\|_{L^{2}(\omega)}+\|\nabla u\|_{L^{2}(\omega)}\right)^{\delta}\|u\|_{H^{1}(Q)}^{1-\delta}
$$

with $\delta=\delta^{\prime} \delta^{\prime \prime}$. Thus, we complete the proof of Lemma (2.2).

We now prove the assertion (4.71).

Let $B_{i}, i=1,2,3$ be three open balls with the properties $B_{1} \subset \subset B_{2} \subset \subset B_{3} \subset \subset Q$. Take $\eta \in C_{0}^{\infty}(Q)$ be valued in $(0,1)$ and $\eta=1$ in $B_{3}$.

Let $y=\eta u$, then $y$ solves

$$
\left\{\begin{array}{lll}
y_{t t}+\sum_{i, j=1}^{n}\left(a^{i j} y_{x_{i}}\right)_{x_{j}}= & \eta_{t t}+2 \eta_{t} u_{t}+\sum_{i, j=1}^{n} a^{i j} \eta_{x_{i} x_{j}} u & \\
& +2 \sum_{i, j=1}^{n} a^{i j} \eta_{x_{i}} u_{x_{j}}+\sum_{i, j=1}^{n} a_{x_{j}}^{i j} \chi_{x_{j}} u, & (x, t) \in Q, \\
\nabla y=y=0 & & (x, t) \in \Sigma .
\end{array}\right.
$$

Denote $P$ the center of $B_{1}$ and let $r(x, t)=\operatorname{dist}^{2}((x, t), P)$, then substitute $\varphi$ by $r$ in $\theta$. By the same argument to the proof of Lemma (2.1), there must be some $\widetilde{\delta}$ with $0<\widetilde{\delta}<1$ such that

$$
\|u\|_{H^{1}\left(B_{2}\right)} \leq C\|u\|_{H^{1}\left(B_{1}\right)}^{\widetilde{\delta}}\|u\|_{H^{1}(Q)}^{1-\widetilde{\delta}} .
$$

For any open ball $B^{\prime} \subset \subset Q$, there is a finite natural number $m$ and two sequences of open balls $\left\{B^{i}\right\}_{i=1}^{n}$ and $\left\{\widetilde{B}^{i}\right\}_{i=1}^{m}$ such that

$$
\left\{\begin{array}{l}
B^{\prime} \subset B^{1}, \quad \widetilde{B}^{i} \subset \subset B^{i} \cap B^{i+1}, \quad i=1, \cdots, m-1, \\
\widetilde{B}^{m} \subset \subset B^{m}, \quad \widetilde{B}^{m} \subset B .
\end{array}\right.
$$


By means of (4.74), there must be a sequence $\left\{\widetilde{\delta}_{i}\right\}_{i=1}^{m}$ with $0<\widetilde{\delta}_{i}<1, i=1,2, \cdots, m$ such that

$$
\begin{aligned}
\|u\|_{H^{1}\left(B^{\prime}\right)} & \leq\|u\|_{H^{1}\left(B^{1}\right)} \leq C\|u\|_{H^{1}\left(\widetilde{B}^{1}\right)}^{\widetilde{\delta}_{1}}\|u\|_{H^{1}(Q)}^{1-\widetilde{\delta}_{1}} \leq C\|u\|_{H^{1}\left(\widetilde{B}^{2}\right)}\|u\|_{H^{1}(Q)}^{1-\widetilde{\delta}_{1}} \\
& \leq C\|u\|_{H^{1}\left(\widetilde{B}^{2}\right)}^{\widetilde{\delta}_{1} \widetilde{\delta}_{2}}\|u\|_{H^{1}(Q)}^{1-\widetilde{\delta}_{1} \widetilde{\delta}_{2}} \leq \cdots \leq C\|u\|_{H^{1}\left(\widetilde{\delta}^{m}\right)}^{\widetilde{\delta}_{2} \ldots \widetilde{\delta}_{m}}\|u\|_{H^{1}(Q)}^{1-\widetilde{\delta}_{1} \widetilde{\delta}_{2} \cdots \widetilde{\delta}_{m}} .
\end{aligned}
$$

Adopting $\widetilde{\widetilde{\delta}}=\widetilde{\delta}_{1} \widetilde{\delta}_{2} \cdots \widetilde{\delta}_{m}$, it follows

$$
\|u\|_{H^{1}\left(B^{\prime}\right)} \leq C\|u\|_{H^{1}(B)}^{\widetilde{\widetilde{\delta}}}\|u\|_{H^{1}(Q)}^{1-\widetilde{\widetilde{\delta}}} \cdot
$$

For that for any $K \subset \subset Q$, there must be a finite subcover of open balls, then from (4.77) we know there is a constant $0<\delta^{\prime \prime}<1$ such that

$$
\|u\|_{H^{1}(K)} \leq C\|u\|_{H^{1}(B)}^{\delta^{\prime \prime}}\|u\|_{H^{1}(Q)}^{1-\delta^{\prime \prime}}
$$

as just claimed.

\section{References}

[1] J. Diestel and J. J. Uhl, Jr., Vector Measures, Math. Surveys, vol. 15, American Mathematical Society, Providence, R.I., 1977.

[2] T.-T. Li, S. Zheng, Y. Tan and W. Shen, Boundary Value Problems with Equivalued Surface and Resistivity Well-Logging, Pitman Research Notes in Mathematics Series, 382, Longman, Harlow, 1998.

[3] G. Lebeau and E. Zuazua, Null-controllability of a system of linear thermoelasticity, Arch. Rational Mech. Anal., 141 (1998), 297-329.

[4] Q. Lü, Observation and Control for Stochastic Partial Differential Equations, Ph.D thesis, School of Mathematics, Sichuan University, Chengdu, 2010.

[5] Q. Lü, Bang-bang principle of time optimal controls and null controllability of fractional order parabolic equations, Acta Math. Sin., 26 (2010), 2377-2386.

[6] J.-L. Lions, Optimal Control of Systems Governed by Partial Differential Equations, Springer-Verlag, Berlin, Heidelberg, New York, 1971.

[7] G. Wang, $L^{\infty}$-null controllability for the heat equation and its application for the time optimal control problem, Siam J. Control Optim., 47 (2008), 1701-1720.

[8] Z. Yin, Null exact controllability of the parabolic equations with equivalued surface boundary condition, J. Appl. Math. Stochastic Anal., 19 (2006), Article ID 62694, 1-10.

[9] Z. Yin, Insensitizing Controls for the Parabolic Equation with Equivalued Surface Boundary Conditions. In submission.

[10] X. Zhang, Solvability of non-linear parabolic boundary value problem with equivalued surface, Math. Methods Appl. Sci., 22 (1999), 259-265. 